Cahiers de philosophie de l'université de

\title{
Totalité : le miroir brisé et la sunopsis. En quête de sources pour la définition IX du Livre des XXIV
}

Anca Vasiliu

\section{(2) OpenEdition}

Journals

Édition électronique

URL : https://journals.openedition.org/cpuc/337

DOI : 10.4000/cpuc.337

ISSN : 2677-6529

Éditeur

Presses universitaires de Caen

Édition imprimée

Date de publication : 31 décembre 2016

Pagination : 13-54

ISBN : 978-2-84133-842-9

ISSN : $1282-6545$

\section{Référence électronique}

Anca Vasiliu, «Totalité : le miroir brisé et la sunopsis. En quête de sources pour la définition IX du Livre des XXIV », Cahiers de philosophie de l'université de Caen [En ligne], 53 | 2016, mis en ligne le 01 février 2019, consulté le 01 février 2023. URL : http://journals.openedition.org/cpuc/337 ; DOI : https:// doi.org/10.4000/cpuc.337

\section{(c) (†) 8}

Creative Commons - Attribution - Pas d'Utilisation Commerciale 4.0 International - CC BY-NC 4.0 https://creativecommons.org/licenses/by-nc/4.0/ 


\section{Totalité : le miroir brisé et la sunopsis. En quête de sources pour la définition IX du Livre des XXIV}

\section{Un anachronisme idéalement pertinent}

Un miroir antique ne se brise pas. Pour cette raison, la métaphore que j'évoque d'entrée de jeu, celle qui nous donne à voir l'entier reflété comme entier dans chaque fragment d'un miroir brisé - métaphore de la présence du «tout» dans le multiple, ou de la totalité comprenant la multiplicité sans que son unité en soit affectée-, ne devrait pas se trouver ad litteram dans un texte antique. Les miroirs antiques sont en argent ou en bronze et, s'ils ont aussi un rôle philosophique, outre leur usage cosmétique, celui-ci est dévolu à la réflexion de ce qui ne se voit pas: à une vue perçante (ỏ $\beta \lambda \varepsilon \dot{\varepsilon \varepsilon}(v)$ ils font apparaître l'âme, ou du moins un reflet du daimôn qui s'y trouve; d'où la fonction «magique» assignée aux miroirs des philosophes. Mais ce rôle épiphanique n'est précisément pas celui pour lequel j'évoque pour commencer le miroir. Il en va ici autrement: bien qu'il soit difficile d'y associer un objet précis, l'idée qu'évoque un anachronique miroir brisé constitue pourtant un thème précis de la pensée antique, un thème illustré quelquefois par cette métaphore du miroir mis en pièces ${ }^{1}$. Un miroir à

1. Plotin, Enn. I, 1 [Traité 53], 8, 17-18 et I, 4 [Traité 46], 10, 17. Ce dernier passage évoque en effet un «miroir fragmenté» ( $\sigma \nu \gamma \kappa \lambda \alpha \sigma \theta \varepsilon \dot{\varepsilon} v \tau(\varsigma)$ pour décrire l'état de l'âme devenue incapable de rendre l'image fidèle de son acte (cette connaissance sensible qui accompagne les actions et que l'on appelle "conscience»), incapacité due à un déséquilibre intérieur, comme une perte de l'harmonie avec le corps et l'intellect. Dans le premier passage, en revanche, il est question, dans le sillage du Timée, de l'âme indivisible et immobile qui se reflète dans les vivants par démultiplication, comme en de nombreux miroirs, générant des reflets qui engendrent le multiple à partir d'un visage singulier ( $\ddot{\omega} \sigma \pi \varepsilon \rho \pi \rho o ́ \sigma \omega \pi$ ov $\dot{\varepsilon} v$

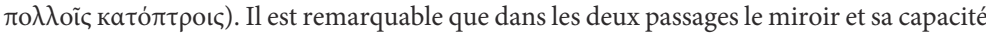
de produire images et reflets sont associés à l'âme et servent à la description de sa nature et de ses fonctions. En ce qui concerne l'usage qui sera fait ici de la métaphore du miroir brisé, précisons qu'il se rapproche plutôt des miroirs multiples illustrant le rapport de 
surface troublée ne rend plus le reflet juste et harmonieux de ce qu'il reçoit ou, au contraire, démultiplie le reflet, faisant apparaître ce qu'une vue directe ne peut pas saisir d'emblée. Plotin, en particulier, semble attentif aux enseignements muets que dispensent les surfaces brillantes dans tous leurs états.

Il s'agit donc d'un moyen iconique ou, mieux encore, d'un modèle hypothétique permettant, sous le mode de l'image, de connaitre in se le tout comme entier infracassable en tant qu'entier, entier infracassable parce que ce tout ne constitue pas seulement un ensemble de parties qui ne tiendraient pas par elles-mêmes, ou que ce tout serait envisagé comme une pluralité réunie par retour sur soi, renversement de la division et conversion à l'unité, mais parce qu'il se définit surtout comme une unité en soi, intégralement homogène à son propre égard et indépendant de ce dont il est «objectivement» composé. Nous ne sommes donc pas loin, en réalité, du miroir qui donnerait à voir ce qui a priori ne se voit pas dans ce qui se voit. Brisé formellement, ce «tout» retrouve l'unité dans chaque fragment, et cette unité retrouvée, bien que formelle dans l'exemple du miroir, indique que le "tout» n'est pas une affaire de quantité et ne se détermine pas par des limites mais par des points de vue. Si chaque partie réfère comme partie à l'entier en tant qu'unité, cette partie comporte alors, nécessairement, une double identité: celle de partie d'un «tout» et celle de la totalité du «tout»- que cette dernière soit la marque du genre et donc la manifestation de l'universel dans le particulier, ou qu'elle soit la manifestation d'un autre lien par lequel l'entier tient en lui-même et peut aussi être saisi comme entier infracassable. Autrement dit, avec les mots d'Aristote, la notion de «tout» (ö入ov) comporte aussi une «totalité qui est

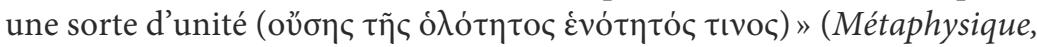
Delta, 1023b 37).

Cette comparaison avec l'effet «magique» du miroir brisé est révélatrice de l'un de ces paradoxes que la pensée antique a circonscrit en cherchant à asseoir sur une base rationnelle ce qui, tenant de l'aporie, est par définition indémontrable more geometrico. Il s'agit, pour le dire avec encore d'autres mots, de définir la possibilité de ce qui ne connaît pas l'impossibilité; en l'occurrence, de saisir ce qui fondamentalement est sans jamais pouvoir être surpris et défini aussi comme possibilité de suspension ou de négation de l'être, bien que ce qui fondamentalement est (ne pouvant pas et jamais ne

l'âme indivisible au mélange du «même» et de l'«autre» et à la division des corps, selon le commentaire de Plotin aux passages 35-36 du Timée. Mais je ne situerai pas cette image dans un contexte cosmologique. Je m'en sers pour illustrer un thème spécifique de la métaphysique, où l'intellect et l'âme interviennent par un acte spécifique de vision. 
pas être), se définit nécessairement comme au-delà de l'être. En effet c'est en tant que tout que ce qui fondamentalement est comprend l'être. Or le «tout» se détermine comme autre qu'être - autre prenant le plus souvent le sens ontologique d'une éminence, d'un dépassement a-topique, et non le sens d'une altérité, d'un contraire ou d'une différence de genre ou de qualité. Ce "tout», qui selon Aristote est une espèce d'unité parce qu'il est plus qu'un ensemble ou autre que la somme de ses parties, sera défini après le Stagirite (nous le verrons plus loin chez Porphyre, par exemple)

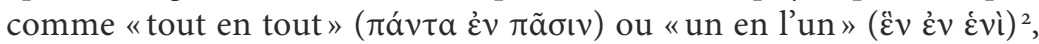
entièrement «tout» en chaque point singulier du «tout»-si du moins on pouvait, mentalement, se figurer un point dans ce qui, parfaitement homogène et solidaire, ne peut que transcender le composé en l'avalant en entier. Solidaire de l'unité propre à sa totalité, ce «tout» ne peut pas se séparer de lui-même pour être reçu: il ne peut être perçu ou pensé autrement qu'en tant qu'entièrement et simultanément «tout», parfaitement et le plus simplement «tout», mais en même temps séparé, orphelin des genres, in-évident, indémontrable et indéfinissable autrement que par des détours - comme lorsqu'il est dit de lui qu'il est, à l'instar de l'un, parfait, seul et simple, sans le définir pour autant. Ou, pour emprunter un exemple à la théorie même de la perception: «chaque partie de l'air, dit

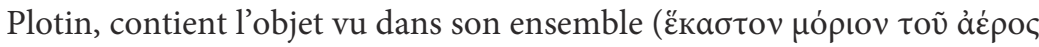

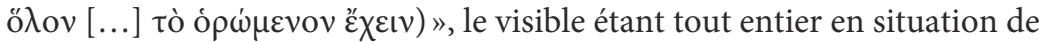
visibilité indépendamment des conditions particulières imposées par tel ou tel angle de la vision, partielle et séquentielle ${ }^{3}$.

L'exemplarité du paradoxe que soulève la possibilité de penser le «tout» comme totalité échappant à l'opération de division et de rassemblement des parties, provient en réalité de la difficile jonction entre d'une part le statut de l'unité en soi, et de l'autre la conception physique, «méréologique», du «tout» qui s'appuie sur l'analyse de la composition et des rapports entre les composants. Au paradoxe de ce «tout» dont la nature serait une sorte de totalité hénotique idéalement homogène, s'enchaîne donc un autre paradoxe, induit par la nature paradoxale de cet «objet» de la réflexion, à savoir qu'à l'égard de ce «tout» irréductible à un ensemble ou à une somme, aucune des catégories n'est opérationnelle pour fournir une définition, hormis la négation systématique de chacune des catégories (substance, qualité, quantité, lieu, temps, relation, etc.), négation qui fournit une définition par défaut. Une exception parmi les catégories semble toutefois se dessiner:

2. Porphyre, Sentences 10 et 44 ; on y reviendra.

3. Enn. IV, 5 [Traité 29] 3, 35-36. Plotin, Traités 27-29, L. Brisson et J.-F. Pradeau (trad.), Paris, Flammarion, 2005, p. 200. 
il s'agit de la relation, mais celle-ci opérerait sous un mode particulier, bien différent de celui qui vise le rapport à l'altérité selon la définition d'Aristote.

Quoi qu'il en soit de la relation (sur la nature de laquelle il faudra revenir), cette impuissance des catégories pourrait alors expliquer le recours constant au reflet, et plus généralement à la connaissance indirecte, lorsque l'on vient à parler $\mathrm{du}$ « tout ». On ne peut en effet saisir la totalité du «tout» qu'en percevant dans chaque partie un reflet de la totalité, puisque la saisie est nécessairement successive, soumise à la temporalité qui divise et à l'entéléchie qui sépare l'accompli de l'accomplissant. Or la totalité, elle, ne tient pas de l'ensemble des parties qui la composent mais de l'unité absolue et parfaitement simple par laquelle la totalité est solidaire avec elle-même au point de se réfléchir ainsi elle-même, toute et entière, dans chaque partie. Ce qui peut recevoir ainsi son reflet, reçoit, en percevant ce reflet qui est un lieu déterminé, la totalité du « tout » indéterminé qui se réfléchit. De cette manière, le reflet lui-même circonscrit l'indétermination foncière de la totalité, sa nature illimitée identifiée au «tout», mais il circonscrit cette nature en tant que reflet de la totalité. La vue peut ainsi saisir (voir) le «tout» dans sa totalité ou, mieux encore, en tant que totalité; elle peut donc le saisir en tant que tenant-ensemble sans lui imposer sa propre limite comme condition d'appréhension par un parcours des parties successives, car, bien que successivement, la vue saisit (voit) en réalité à chaque fois tout.

Mais dans un domaine apparemment inaccessible aux moyens rationnels, de quelle sorte de vue s'agit-il ? Plus précisément, la vue court-circuite-t-elle la raison ou simplement prend-elle la relève comme un moyen alternatif au langage, mais toujours intégré au domaine de la raison? La condition de possibilité de la réception ne peut que se trouver inscrite dans ce qui se donne, non dans ce / celui qui reçoit; elle ne peut pas provenir de la projection réceptive ou théorique et ne peut pas limiter par conséquent ce qui se donne à constituer un objet de l'expérience, soumis à la condition «subjective» réceptrice, sensitive et intellective, de ce/celui qui le reçoit. L'acte n'appartient-il pas alors à ce / celui qui l'actualise, comme si cet acte provenait d'ailleurs? Force est d'admettre que c'est en effet par l'accidentel que l'universel se dévoile dans ce cas, si on considère, à partir d'Aristote, que l'universel est en effet implicite à la totalité de ce «tout » infracassable qui est une sorte d'unité. Par l'accidentel, par le sensible, par le matériel - dont le miroir est d'ailleurs considéré comme étant le signe ou le symbole - s'actualise en effet, selon la nature du récepteur, ce qui n'est pas dans la nature de celui-ci. Car ce qui se dévoile ainsi n'est précisément pas de l'ordre de l'accidentel; ce n'est là qu'une condition par laquelle ce qui ne connait jamais l'accidentel se laisse approcher par des moyens opposés à ce qu'ils révèlent. Ces moyens, contraires en apparence, sont de 
facto eux-mêmes inscrits dans ce qui se révèle par leur truchement, et la condition même de leur possibilité y est inscrite; cette dernière se trouve comme condition dans ce qui peut s'actualiser et s'actualise à la mesure exacte de ce qui en provient.

Le processus est donc plus complexe qu'un simple renversement d'opposés avec un dépassement conséquent de la différence. Au-delà du paradoxe, le recours au reflet et donc à la vue, au visible et à l'image, se trouve néanmoins infléchi par une compréhension restrictive imposée à ces notions mêmes. La vue n'est pas la vue sensible comme telle mais une modalité homonyme de la saisie intellective, de même que le visible n'est pas la qualité de l'étant corporel mais désigne plutôt la condition d'intelligibilité de l'intelligible dans sa nature même. L'image, à son tour, se confond avec ce que peut fournir l'idée du reflet, tel le reflet de l'intellection reçu par / dans l'âme. Ce n'est donc pas une image destinée aux yeux, une image qui se laisse parcourir par une vue qui chemine, mais cette image dont un seul éclair suffit au regard perçant pour saisir d'un simple coup d'œil ce qui vient de se révéler dans son champ. Il convient donc de circonscrire cet usage particulier de la vision et de le définir en rapport avec le contexte éminemment théorique et abstrait de l'intellection. Il faut donc le situer dans le registre des intelligibles partant d'un constat: à savoir que la perception successive dans le temps, dénoncée comme un moyen qui ne convient pas à la totalité puisqu'elle introduit une division dans l'unité, n'est de fait pas une perception spécifique à l'image, mais semble plutôt empruntée au caractère séquentiel propre au langage; c'est comme si on imposait à la saisie visuelle le modèle du parcours propre à la «lecture», alors que cette image intellective, par rapport à la construction discursive du langage, se caractérise au contraire par l'immédiateté de la saisie d'ensemble de ce qu'elle déploie comme visibilité, même si cette visibilité ne comporte pas une étendue égale à la totalité d'un ensemble. Cette remarque pourrait fournir un indice des sources textuelles hétérogènes qui se croisent dans cet usage préférentiel du reflet, de l'image et de la vue aux dépens des catégories analytiques, dites "de l'être».

Retenons donc les aspects suivants parmi les questions que soulève la détermination $\mathrm{du}$ "tout» en tant que totalité, c'est-à-dire du "tout» sous l'espèce de l'unité: (1) la nécessité de trouver un moyen adéquat de connaissance indirecte; (2) la référence préférentielle à la possibilité de saisie perceptive, et précisément visuelle, qui répond à l'injonction comprise dans le premier aspect saisi; enfin, (3) le détournement de celle-ci au profit d'un modèle impropre, en réalité, à la saisie visuelle et à l'image, à savoir le modèle séquentiel de la discursivité. Distinct du paradigme de la vision, le recours au modèle discursif sans écarter toutefois le vocabulaire de la vue 
et du regard pourrait relever de la nécessité (a) de justifier le paradoxe par la voie d'un recours impropre en apparence au but visé, (b) de souligner que la totalité sous l'espèce de l'unité comporte une réalité en soi et ne se dit pas ainsi par analogie, et (c) d'indiquer que le passage par l'accidentel signifie, du moins de manière référentielle, que la totalité (holotês) en tant qu'unité ne se confond pas avec l'universel (pantotês) mais constitue sa transcendance.

\section{Un-unité, Tout-totalité, Dieu-déité}

Ces couples de mots, outre leur propre charge sémantique, évoquent par la répétition la possibilité de concevoir une approche à la fois directe et dérivée de ce qui ne connaît aucune séparation de soi et ne comporte aucune différence dans l'intimité de son identité et de sa nature. C'est la répétition qui permet d'ouvrir un chemin là où la racine commune des mots ne laissait entendre qu'une identité stricte et fermée. La répétition permet ainsi d'envisager une relation, même s'il ne s'agit que d'une relation paradoxale puisque sans altérité, une relation autoréférentielle qui ne peut ni produire ni indiquer une modification substantielle ou formelle conséquente à l'approche par la pensée, mais peut du moins en attester la possibilité et en esquisser la forme propre: la réflexion, relation spécifique destinée à servir, en particulier dans ces cas, de condition pour l'approche rationnelle. En d'autres termes: la réflexion conditionne, dans ces trois cas, l'acte de penser. La répétition, signalant la possibilité d'une relation, crée en outre un lien. Même s'il ne s'agit, là aussi, que d'un lien paradoxal, réduit à relier le même au même par une sorte de dérivation ou de dédoublement d'un seul et unique «soi» signifié par les deux termes, ce lien endosse néanmoins un rôle déterminant lorsqu'on doit réduire la relation et donner un statut unique à la chose deux fois nommée. Peut-on qualifier ce lien de paronymique (car la synonymie et l'homonymie sont d'emblée exclues) ? Quelle serait en outre la différence signalée par un lien a priori structurel, si ces paires de mots, désignant chacune une identité sans fracture, nomment de surcroît la réalité essentielle selon laquelle cette identité s'accomplit, bien que la perfection de celle-ci consiste précisément à ne disposer d'aucune réalité ontologique propre - si tant est que l'Un, le Tout et Dieu se définissent chacun par une forme spécifique de transcendance face à ce qui ne serait qu'immanent à l'être et connaissable par les catégories?

La réponse obvie se résume à définir l'unité comme propre à la nature de l'Un, la totalité comme propre à la nature du Tout, la déité comme propre à la nature d'un ou de Dieu. Mais, à cette recherche de précision à 
l'égard du propre d'une chose par une relation réductive de sa nature à son actualisation ou à sa réalité essentielle, s'ajoute le sens réflexif inhérent à cette autoqualification solipsiste de la nature par le propre, sens réflexif qui comprend le lien (ou la non-séparation) et porte, avec celui-ci, la charge sémantique de l'accomplissement. C'est bien cette visée entéléchique du réflexif que semble illustrer totalité à l'égard du Tout, par-dessus la détermination du Tout comme «ensemble indivisible» ou, avec les mots de

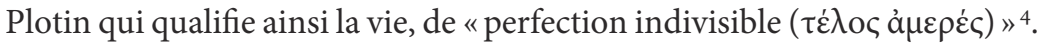
Or ce sens réflexif de la réalisation complète, parfaite pour autant qu'elle comporte une abstraction ou une transcendance et qu'elle le manifeste sous le mode double d'une donation et d'un retour sur soi, est indicatif de la démarche propre de l'âme rationnelle à l'égard de sa nature. Cette démarche de l'âme se distingue de la démarche purement intellective qui est, elle, dans une relation réductive à l'identité, du «tout au tout exactement», parce que l'intellection s'identifie à l'objet intelligé en en faisant le propre entier et simultané de l'intellect. Comment comprendre néanmoins ce sens réflexif de réalisation dans le cas de Dieu ou de l'Un, si ce sens correspond en réalité à la démarche de l'âme et signifie à la fois son acte cognitif et sa nature? Ont-ils, eux aussi, Dieu ou l'Un, besoin de la déité ou de l'unité pour s'accomplir comme perfection par donation et retour sur soi? Certes, non. En revanche, la réflexion et le lien qui se réalisent à travers déité ou unité, brisent ainsi la coque de la monade absolue. Ici encore, comme dans le cas du Tout-totalité, c'est la condition du modèle réflexif propre à l'âme rationnelle qui semble en assurer l'accès. L'exemple du rapport entre totalité et Tout sert en effet à mettre en évidence précisément ce processus. Il est d'ailleurs analysé de manière dialectique par Platon dans le Théétète (204a1 sq. à propos de la science par division, somme du tout et différence par rapport à la totalité) et dans le Parménide (145b8-e5 et 157 C3-158a 8 , au sujet de la définition de l'un à partir du rapport entre tout et parties vu comme une relation d'exclusion ou d'inclusion entre le soi et l'autre), Aristote étant à l'évidence l'héritier direct de cette analyse en ces termes mêmes dans la Métaphysique (voir infra). Et c'est bien sur le modèle de ce processus d'abstraction et de dédoublement réflexif que déité et unité peuvent alors être envisagées comme des conditions de possibilité pour concevoir Dieu ou l'Un, sans pour autant réduire ces derniers à de purs «objets de pensée». L'exemple paradigmatique du Tout-totalitén'est cependant pas exempt d'écueils; son vocabulaire évoque la pluralité, et une connivence avec la pensée de l'universel se laisse soupçonner, en qualifiant

4. Enn. III, 7 [Traité 45], 3, 19. J’y reviendrai. 
son usage de moyen hétérodoxe pour une théologie stricte du Dieu un et tout en même temps.

Analysons brièvement deux mises en situation où totalité se détermine à la fois en lien et par différence à l'égard du «tout», deux passages, bien déterminés contextuellement, et qui ont d'ailleurs nourri les propos tenus jusqu'à présent. L'un est tiré d'un ouvrage latin où apparaitt (serait-ce pour la première fois?) le terme totalitas. L'autre, nous l'extrayons d'un livre grec

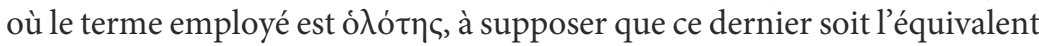

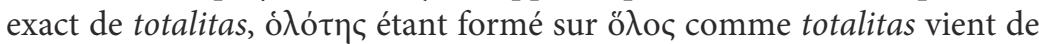
totus - bien que la différence de ces termes par rapport à $\pi \tilde{a} \varsigma, \pi \tilde{a} v, \pi \alpha ́ v \tau \omega \varsigma$,

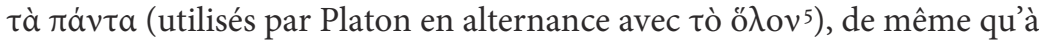
l'égard du classique omnis ou encore du tardif totaliter (Cassiodore) et du médiéval totalis (Oresme), ne soit pas aussi claire que l'on puisse justifier d'emblée l'exclusion d'autres contextes où le «tout» se dit et se définit à travers ces derniers termes. Au fond, sous la notion de «totalité» c'est toujours le jeu du tout, de l'universel et de la possibilité d'un accès à l'unicité que mène la pensée à travers les mots. Mais résumons-nous à ces deux contextes: l'un proprement aristotélicien, l'autre tardo-platonicien (qu'il soit encore antique ou proprement médiéval). Ces contextes, aussi éloignés soient-ils, l'un matriciel, l'autre dans l'apogée de l'assertion emblématique, sont néanmoins riches de bon nombre d'aspects propres à ce jeu du tout, de l'universel et du singulier, jeu qui correspond à la recherche depuis Platon d'une solution dialectique à l'opposition trop franche entre l'un et le multiple ${ }^{6}$.

5. Pour la difficile distinction entre ta panta et to holon, la «somme» et le «tout», ou «l'ensemble des parties» et la "totalité», il faudrait revenir non seulement aux apories de l'un et du multiple exposées dans le Parménide (dans les passages cités supra), mais aussi à la discussion entre Socrate et Théétète censée déterminer de manière dialectique, par identité et différence à partir de cet exemple précis, les conditions de la connaissance (Théétète, 204a1-205a8). Nous avons toutefois privilégié, pour des raisons détaillées plus loin, l'analyse d'un autre contexte philosophique où le rapport tout-totalité se dit et est défini à travers une terminologie fondée sur une racine commune: holos-holotês.

6. Au fond, la situation pourrait se résumer ainsi. Soit nous restons, avec le Parménide, dans

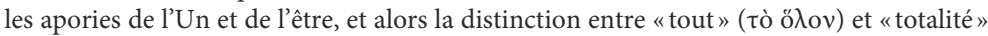
( $\tau \grave{\alpha} \pi \alpha \dot{v} v \tau)$, se réduit à voir d'un côté l'un en tant que "tout» comme en-autre que soi,

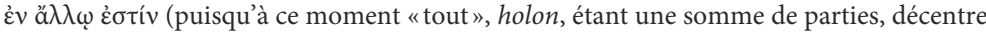
nécessairement l'un de lui-même), et de l'autre côté l'un en tant que «totalité » comme

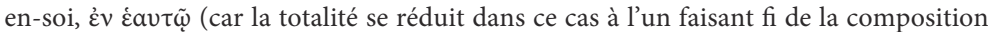
des parties); dans ce cas l'un est à la fois même et autre (c'est la conclusion du passage 145a1-e8). Soit nous sortons la question du domaine strict de l'être et nous la situons, avec le Théétète, et davantage avec Aristote, sur le plan d'une science qui n'est pas rivée aux genres ou aux catégories de l'être puisqu'elle propose d'aborder la question du «tout» et de la «totalité», et donc de l'un et du multiple, par les moyens de la logique. Nous assistons alors à une opération de repli entre «tout» et «totalité», repli sur la distinction entre un 
Prenons à rebours le chemin présumé des textes. Une lecture ancienne (tardo-antique ou médiévale) attire parfois l'attention sur des aspects auxquels d'emblée nous ne sommes pas sensibles lorsqu'on reprend, inlassablement, les textes-sources. Dans le Livre des XXIV philosophes (selon le titre traditionnel donné par Maître Eckhart) ou des XXIV définitions de Dieu (selon le contenu du recueil), le terme latin totalitas vient déterminer dans le commentaire de la définition IX le lien entre la nature divine et le « tout » 7 . Le style elliptique des définitions donne force à l'assertion autant qu'il sème un certain trouble dans la pensée, ou du moins un certain désir d'éclaircissement qu'exprime, presque tout aussi brièvement, le commentaire de la définition:

« tout» comme ensemble (divisible) et une " totalité » sous l'espèce de l'un en tant qu'unité, mais avec la nécessité de retrouver cette «totalité» entière dans l'unicité de chaque partie et même dans l'absence d'une partie. On peut saisir cette "totalité» reflétée dans chaque partie soit comme l'universel du genre soit comme la forme. La seconde démarche, qui comprend les conditions de possibilité de la connaissance, est privilégiée aussi bien par Plotin et Porphyre, que par les approches rationnelles du Dieu-Monos dans la théologie chrétienne.

7. Définition IX, in Livre des XXIV philosophes, F. Hudry (éd. et trad.), Paris, Vrin, 2009, reprise avec ajouts et modifications de l'édition et traduction, Grenoble, J. Million, 1989. Nous n'entrons pas ici dans les questions controversées de la datation de ce texte que la plupart des historiographes considèrent comme un texte médiéval (de la fin du XII ${ }^{e}$ siècle) et que F. Hudry, s'appuyant sur une minutieuse étude comparative codicologique, philosophique et philologique, situe au $\mathrm{IV}^{\mathrm{e}}$ siècle. Notre enjeu étant théorique, nous nous contentons de formuler quelques hypothèses sur différents aspects qui sont réunis dans le commentaire de cette définition et suggérons quelques parallèles avec des passages de référence dans les textes de la pensée antique classique et tardive, situés en amont ou au $\mathrm{IV}^{\mathrm{e}}$ siècle même, en espérant que ces quelques parallèles pourront nourrir la réflexion des historiens des textes et des doctrines en quête d'arguments pour la datation de cet important recueil. Quoi qu'il en soit, tardo-antique, provenant de la même ambiance philosophique que les écrits de Marius Victorinus, ou médiéval, relevant de ce que l'on appelle le courant de l'avicennisme latin, ce recueil de définitions théologiques comporte clairement des éléments de philosophie antique classique passés au crible de la pensée de Plotin et de Porphyre. Ce sont certains de ces éléments que nous mettons plus loin en lumière. Signalons aussi un article récent de F. Hudry sur d'autres aspects concordants entre ce recueil et des problématiques philosophiques propres au platonisme tardif: «L'id ipsum de Marius Victorinus à Porphyre», in Autos, idipsum. Aspects de l'identité d'Homère à Augustin, D. Doucet et I. Koch (dir.), Aix-en-Provence - Marseille, Presses universitaires de Provence, 2014, p.101-113. Dans cet article, le Livre des XXIV est explicitement attribué à Victorinus. Les autres principaux éditeurs et exégètes de ce texte penchent nettement vers une datation médiévale. Parmi les plus récents voir: Il libro dei ventiquattro filosofi, P. Lucentini (trad.), Milan, Adelphi, 1999; Was ist Gott? Das Buch der 24 Philosophen, K. Flasch (trad.), Munich, Beck, 2011; Ce este Dumnezeu? Cartea celor 24 de filosofi, D. Coman (trad.), Oradea, Ratio et Revelatio, 2015 ; Z. Kaluza, «"Comme une branche d'amandier en fleurs". Dieu dans le Liber viginti quattuor philosophorum", in Hermetism from Late Antiquity to Humanism, P. Lucentini, I. Pari et V. P. Compagni (éd.), Turnhout, Brepols, 2003, p.99-127. 
DEUS EST SOLI [/ CUI SOLI] PRAESENS QUICQUID CUIUS TEMPORIS EST.

Haec definitio est secundum formam.

Totum quidem uno aspectu omnes partes videt, pars vero totum non videt nisi diversis respectibus et successivis. Propter hoc deitas successivorum totalitas. Unde eius intuitus unicus est et non consequenter factus.

\section{DIEU EST, POUR LUI SEUL, LE PRÉSENT DE TOUT CE QUI APPARTIENT} AU TEMPS.

Cette définition est selon la forme.

Le tout en vérité voit toutes les parties d'un seul coup d'œil, mais la partie ne voit le tout que sous des aspects successifs et divers. C'est pourquoi la nature divine est la totalité des éléments successifs. Par conséquent, elle a un regard unique, et non façonné en allant d'une chose à l'autre ${ }^{8}$.

Dans un tel type d'écriture, relevant du genre sentences ou définitions et commentaires brefs, pratiquement tous les mots comptent et sont lourds à la fois de sens et d'échos à des textes de référence ${ }^{9}$. L'auteur du recueil de sentences commentées n'hésite pas, d'ailleurs, à faire précéder la série des définitions d'un prologue qui annonce le genre en définissant en quelque sorte la définition et en fixant du coup les règles du jeu. Dans une assemblée de sages, sans doute fictive, chacun à son tour propose « une explication appropriée sur Dieu sous forme de définition (interpretationes sub definitione), en vue d'établir d'un commun accord, à partir de leur définitions propres, quelque chose de certain sur lui ${ }^{10}$. Une première définition - Dieu en tant que monade (Monos) - est ensuite proposée secundum imaginationem primae causae (selon le mode de la cause première, mode propre à la faculté de représentation ou d'imagination) et toutes les autres définitions suivent en respectant la même structure de présentation à quatre temps : (i) énoncé de la définition proprement dite; (ii) indication modale (secundum... essentiae, ou formam, ou modum imaginandi, ou speciem, ou

8. Livre des XXIV philosophes, F. Hudry (trad.), Grenoble, J. Million, 1989, p.168-169. Pour la définition, je me permets de proposer une variante de traduction: «Dieu est celui pour qui est présent tout ce qui appartient au temps».

9. Les études de K. Flasch et de D. Coman, citées supra (note 7), sont particulièrement attentives à la présentation formelle propre à ce recueil, en y voyant à la fois les sources d'inspiration possibles (Cicéron, Porphyre, Boèce) et un éventuel indice pour la datation et la contextualisation du texte dans le milieu théologique latin du tournant des XII et $\mathrm{XIII}^{\mathrm{e}}$ siècles, notamment en rapport avec les milieux chartrain et porrétain, et avec plusieurs écrits d'Alain de Lille (Règles de théologie, Summa Quoniam homines, Sermon sur la sphère intelligible) dont certaines propositions et gloses du Liber sont particulièrement proches.

10. «[...] singuli de Deo proprias proponerent interpretationes sub definitione, ut ex propriis definitionibus certum id de Deo communi consensu statuerent », Livre des XXIV..., p.148-149. 
rationes diversas ipsius essentiae deitatis, ou encore sub relatione, per effectum etc.); (iii) glose brève; (iv) parallèle ou comparaison - à cette réserve près que la quatrième approche n'intervient pas régulièrement ${ }^{11}$. La définition IX, censée présenter Dieu à travers les notions de «tout» et de «totalité», obéit, elle, à la règle de la définition «selon la forme » et comporte trois des quatre approches, définition, mode, glose brève, cette dernière donnant toutefois l'impression d'intégrer aussi une sorte de parallèle ou de comparaison.

Voyons d'abord les raisons du mode choisi. La définition IX installe Dieu dans le rapport au temps. Dieu est en dehors du temps mais, s'il faut le définir à l'égard du temps et nécessairement dans le temps propre à la séquentialité d'une définition, ici sous l'espèce de la forme, secundum formam, Dieu est alors le "présent de tout ce qui est [dans] le temps». Le "présent» (praesens), selon la catégorie temporelle, n'objectivise pas Dieu, n'en fait pas l'objet hic \& nunc d'une parousia, mais signale que l'unicité temporelle du présent peut être élevée au rang d'unicité parfaitement identique ou égale à elle-même en tout ce qui est, et que cette compréhension, «sous l'espèce de la forme», s'exemptant à la fois du temps et de l'être, correspond au statut même assigné à Dieu. La non-temporalité de Dieu le qualifie en tant que présence présente, actuelle, solidaire et unique, identifiée à chaque unité temporelle comprise comme "présent». De manière aporétique, par suspension ou retrait, la négation engendrerait toutefois une réalité, voire serait elle-même l'accomplissement de cette réalité. Passé et devenir abolis, le présent devient, dans ces conditions, présent illimité ou perpétuité pour celui qui est éternel. Or, à cette perpétuité du présent, encore distincte de l'éternité, répond / correspond, dans la partie glosée de la définition, la totalité. Mais suivons une à une les étapes serrées du raisonnement qui conduit aux formulations resserrées de l'assertion et du commentaire.

À son compte, le «tout saisit» (totum videt) sous « un seul aspect» (uno aspectu) toutes les parties (omnes partes). Les parties, en revanche, «ne saisissent le tout» (totum non videt) que dans le multiple se présentant de manière successive et comme divers (diversis... et successivis) ${ }^{12}$. Il est vrai que le multiple n'est pas nommé comme tel, mais il surgit à la fois du pluriel indiqué des parties et de la double qualification de «divers» et de «successif» donnée par l'acte de la saisie, ce qui indique, outre la pluralité, une non-identité essentielle entre les parties. Le «tout» n'est donc «un»

11. On peut y voir, outre l'appel aux catégories, l'adaptation d'une structure rhétorique classique, mais une analyse de la composition du point de vue strictement rhétorique n'a pas encore été entreprise, à ma connaissance.

12. Volontairement, je ne reprends pas exactement la traduction de F. Hudry mais la transposition à la lettre du texte latin, dans laquelle j'insère, au sens médiéval, une traductioninterprétation de la démarche à laquelle obéit, me semble-t-il, la définition. 
que de son côté; il est en revanche multiple à partir de ce dont il constitue l'ensemble, à savoir, à partir de ses parties. Le «tout» n'est donc pas de manière univoque une unité solidaire et homogène en soi, mais bien, de manière équivoque, il est un et composé en même temps. Cependant, cette perspective double ne génère pas une contradiction, car elle est la conséquence d'une définition secundum formam et d'une analyse de la saisie, appelée ici par le terme correspondant à la faculté visuelle (videt). Seule la réciprocité naturelle entre la vue et le visible se trouve infléchie par l'asymétrie de cette perspective double selon laquelle chacun «voit» la même chose autrement: d'une part le tout-un, de l'autre le tout-ensemblede-parties diverses et successives. Mais laissons pour l'instant de côté cet aspect, sur lequel il faudra revenir plus longuement à la recherche d'une justification pour ce choix explicite de la vue dans une définition a priori opposée au mélange des registres, et concentrons-nous d'abord sur les déterminants du «tout».

Totum correspond en effet à une somme de parties. «Tout» désigne donc un ensemble et sa nature est bien celle d'un composé. On peut considérer que le temps lui-même est composé de ce à partir de quoi il se déploie tout en y participant (quicquid cuius temporis est). Toutefois, si le temps est luimême un «tout», alors ce «tout» comporte aussi à l'égard du temps une possibilité d'échapper à cette nature composite. Il y a en effet dans le temps quelque chose qui transcende le temps, à savoir ce qui est perpétuellement présent et ne connaît ni le devenir ni la division des parties puisqu'il est seul et unique en tant que présent. C'est sur cette aporie, sur cette vérité indémontrable que s'appuie la formulation de la IX définition de Dieu (Deus est soli praesens...). On voudrait, certes, pouvoir mieux cerner cette notion de "présent», cette sorte d'actualité de Dieu conjuguée au présent de «ce qui est dans le temps", mais la définition lapidaire ne donne qu'une seule indication: présent à l'égard de ce qui est dans le temps. Le «présent» est donc utilisé pour nier toute détermination temporelle dont Dieu pourrait ici faire l'objet: le "présent» s'exempte en effet du temps et constitue à l'égard du temps une actualité qui se tient hors du temps, comme si le temps lui-même s'autotranscendait par/dans le présent.

Saisies depuis la position prééminente du totum, les parties sont toutes simultanément présentes. L'approche «méréologique» du «tout»par rapport aux parties illustre ce que signifie l'abolition du temps dans la définition de Dieu comme "présent», car, si le temps est formellement déterminé par la succession, il ne peut qu'être opposé à la simultanéité existentielle de toutes les parties en présence, qu'elles soient passées ou futures. Le présent temporel est donc, de facto, irréductible au temps. Mais, une seconde conséquence apparait aussi depuis cette position tenue par le 
«tout», à savoir que le multiple non plus n'existe pas, car il est dit dans le commentaire de la définition que le «tout» embrasse de son regard tout simultanément : totum... omnes partes videt. Il s'ensuit que Dieu, pour qui est présent tout ce qui est dans le temps, n'est pas lui-même dans le temps et n'est pas, du même coup, multiple. Dieu arrache en effet le moment présent au temps, comme le regard unique et global, en embrassant le «tout-entier», extrait ainsi le «tout» de la multiplicité d'un ensemble composé de parties. Jusqu'ici le parallèle correspond au contenu de la définition de Dieu (i). Mais en réalité, la glose brève (iii), qui fait suite à l'indication modale (ii), ne s'intéresse plus à Dieu mais à la «déité» (deitas) ${ }^{13}$, au propre de celle-ci, ainsi qu'à la modalité d'appréhender le «tout» soit comme un, soit dans la succession de ses parties différentes, donc comme ensemble.

Comment faire alors le lien entre la définition de Dieu pour qui est présent tout ce qui appartient au temps et la glose qui stipule la double saisie du «tout» et définit la déité en relation avec cette double saisie? C'est la totalité (totalitas) qui répond précisément à cette interrogation, mais, en répondant, la totalité opère une mutation: elle déplace la définition de Dieu sur le plan d'une définition de la «nature» de Dieu, donc, depuis la quiddité vers la propriété. Le propre de la déité s’identifie à la totalité des choses successives dont est composé le «tout» en tant qu'ensemble des parties. Propter hoc deitas successivorum totalitas. Donc la totalité répond à la nécessité de définir la déité, de la même manière que le présent, perpétuellement unique et seul, correspond à Dieu face à ce qui est dans le temps. Apparaît ainsi entre "totalité» et "présent singulier» un trait commun négatif, à savoir que ni la totalité ne recoupe le statut propre du «tout», ni le présent n'appartient en propre au temps, mais chacun transcende ce par rapport à quoi il se détermine en s'en extrayant: la totalité face à la somme des choses successives, le présent comme perpétuité du moment unique par rapport aux choses qui sont dans le temps. Cette description correspond à la manière dont Dieu lui-même transcende, autant par la quiddité que par la propriété, ce qu'il ne peut déterminer qu'en s'en extrayant: le temps et le tout-entier de la pluralité des étants. Il ne faut toutefois pas prendre ce parallélisme pour une analogie, au sens technique de cette figure. Rien dans cette articulation entre définition et glose n'indique une construction analogique, de même que rien n'indique non plus une procession et une remontée ou explicitement un principe et sa manifestation causale. Il s'agit

13. F. Hudry y voit un terme spécifiquement chrétien parce qu'il est employé par Arnobe, Hilaire de Poitiers, Victorinus, Augustin (Livre des XXIV..., p. 159, note b). Mais pourquoi ne serait-il pas la traduction «sans étiquette» de la divinité ( $\theta \varepsilon$ เó $\eta\rceil \varsigma)$ ou de la nature divine $(\theta \varepsilon \tilde{\alpha} \alpha, \theta \varepsilon \tilde{\varepsilon}$ ○६), termes du vocabulaire courant des théologies? 
plutôt de montrer l'impossibilité d'établir une inférence au sujet des paires conceptuelles de l'être ou de la nature impliquées dans la définition - un / seul-unité, tout-totalité, dieu-déité, présent / éternel-perpétuité -, et de proposer en échange l'usage d'un moyen d'appréhension spécifique, dont l'exercice apparaît comme étant le plus idoine à la saisie de ce qui $a$ priori est sans possibilité de saisie ou de connaissance.

De l'unicité singulière du présent, comme de la totalité qui est, elle aussi, toujours une en même temps qu'expression de l'unité par rapport à la multiplicité naturelle des choses successives, il n'y a en effet ni genre, ni espèce, ni catégorie adaptée, ni forme universelle. Ce n'est pourtant pas de la négation de celles-ci dont se sert l'auteur du Liber mais d'un moyen différent, désigné comme vue d'ensemble, tel un coup d'œil synoptique. Ce moyen est vraisemblablement emprunté à la philosophie de Platon, mais non à la théorie des formes, au Parménide pour ne pas le nommer, piste que semble pourtant indiquer la précision modale (secundum formam) qui fait suite à la définition.

Avant de revenir sur ce moyen spécifique deux fois évoqué dans le commentaire de la définition IX par une terminologie empruntée à la vue (videt, intuitus), rappelons et analysons aussi, brièvement, l'un des textes grecs fondateurs pour la définition du «tout» et précisément de la totalité par rapport au «tout» et de l'unité par rapport à l'«un».

Un chapitre entier du livre Delta de la Métaphysique y est consacré, un livre que certains de ses lecteurs de l'Antiquité tardive considéraient comme une introduction à la Métaphysique et comme une sorte d'initiation aux Catégories. Lisons ce large extrait du chapitre 26:

Un tout s'entend (ö $\lambda$ ov $\lambda \dot{\varepsilon} \gamma \varepsilon \tau \alpha$ ) de ce à quoi ne manque aucune des parties

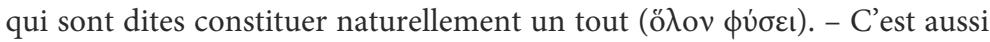

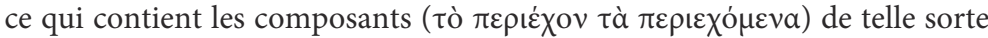

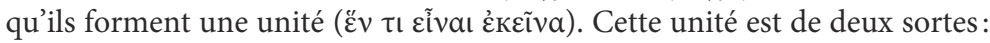

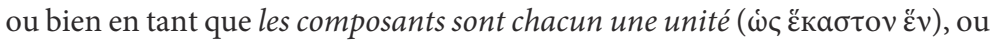

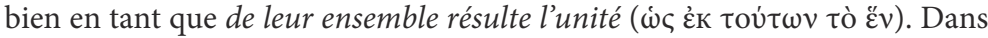

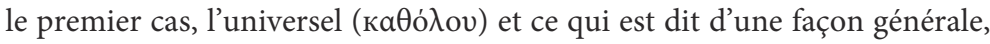

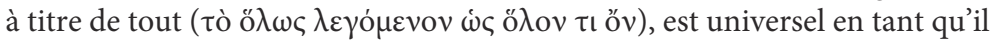
embrasse une multiplicité d'êtres, par le fait qu'il est prédicat des composants

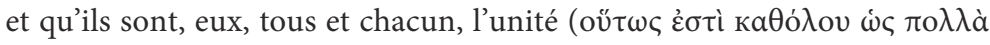

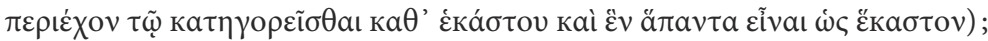
par exemple, homme, cheval, dieu ( $\theta \varepsilon \dot{v} v$ ) [sont un], parce qu'ils sont tous des

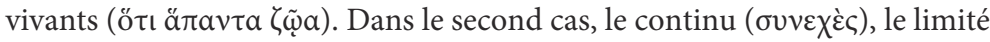

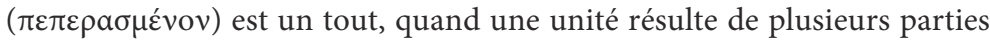

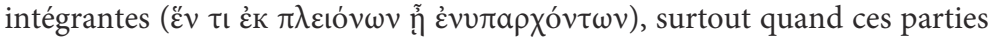
sont seulement en puissance ( $\delta v v a ́ \mu \varepsilon ı)$, et, à défaut, même quand elles sont en 


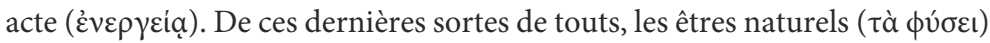
sont plus véritablement tout que les artifacts ( $\eta \hat{\tau} \tau \dot{\varepsilon} \chi \eta \eta \eta)$, comme nous avons

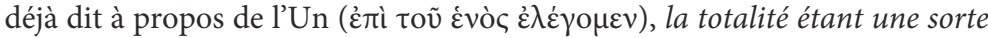

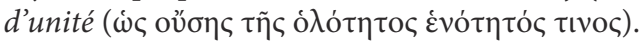

En outre, des quantités ayant un commencement, un milieu et une fin, celles dans lesquelles la position des parties est indifférente sont appelées un total $(\pi \tilde{\alpha} v)$ et les autres, un tout (ö $\lambda \circ v)$; celles qui peuvent réunir les deux caractères

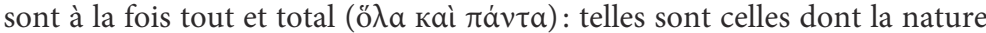
( $\phi \dot{\sigma ı \varsigma \varsigma) ~ r e s t e ~ l a ~ m e ̂ m e ~ a p r e ̀ s ~ l e ~ d e ́ p l a c e m e n t ~ d e s ~ p a r t i e s, ~ m a i s ~ d o n t ~ l a ~ f i g u r e ~}$

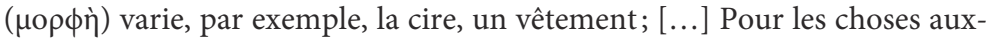

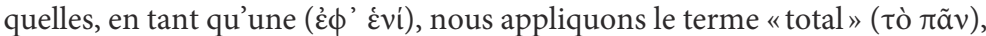

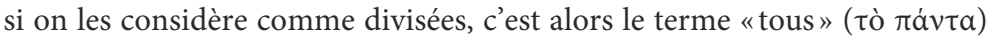

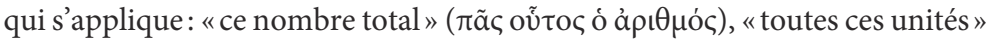

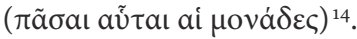

Il n'est pas difficile de saisir dans ce texte bien connu les raisonnements et les éléments de langage qui rappellent les questions analysées dans la définition IX du Livre des XXIV. Entendons-nous bien, toutefois: il ne s'agit pas d'établir une filiation entre ces textes, mais de saisir des mécanismes de réflexion qui ont pu conduire aux formulations aporétiques et aux commentaires lapidaires du Liber, ou du moins, qui ont pu les inspirer. Les proximités ou les confluences, de même que les divergences ad litteram, n'ont pas nécessairement pour vocation de prouver un lien textuel direct, comme de cause à effet. Elles éclairent en revanche la base d'une connaissance non nécessairement du texte aristotélicien lui-même, mais des réflexions thématiques nourries par les textes classiques et d'un mode de formuler la pensée entretenu par le ressourcement continu qu'assure le travail des commentateurs. Du reste, trouver la preuve des sources (ou des origines) est souvent œuvre du hasard.

Remarquons d'emblée que dans la définition aristotélicienne, comme dans le commentaire de la définition du Liber, le «tout» est indissociable de l' «un », de même que la «totalité » est inséparable de l' «unité ». Ces couples conceptuels, un-tout et unité-totalité, désignent ce qu'on pourrait appeler,

14. Métaphysique, Delta, 26, 1023b 27-1024a 11, J. Tricot (trad.) [traduction légèrement modifiée], Paris, Vrin, 200o, p. 214-215; pour le grec, éd. Loeb, 1933. La récente traduction de R. Bodéüs et A. Stevens (Métaphysique, Delta, Paris, Vrin, 2014) ne comporte pas de différences notables, dans ce chapitre, avec la traduction de Tricot. Précisons par ailleurs, s'il le faut encore, que le but n'étant pas une étude de la conception «méréologique» d'Aristote mais une analyse des usages de la «méréologie» en contexte théologique, il serait indispensable d'aborder ici également les chapitres 10 et 11 du livre Zeta où la question des parties est posée dans une perspective de définition ousiologique et où l'intérêt du philosophe est mobilisé par le statut des formes et des universaux. 
ici encore, des «identités différées $»^{15}$. Ce sont des identités temporairement imparfaites, des items non entièrement identiques puisqu'ils comportent une différence de l'ordre d'un décalage voué à se résorber au terme d'un perfectionnement qui, bien que spécifique à chacune des entités relevant d'un ensemble, est simultanément propre à l'homogénéité foncière de l'ensemble lui-même. Un et tout tendent en effet, chacun, à accomplir l'unité et la totalité au terme d'une identité réalisée en tant qu'entéléchie de soi-même. S'actualisant par autonégation et s'accomplissant comme résorption, la différence est bien celle qui réduit ainsi la pluralité induite à partir de chacune des identités à un seul et même item identique, lequel ne s'identifie pas à l'universel du genre mais le transcende en transcendant le caractère composé d'un ensemble pour donner unité à l'« un » et conférer la totalité au «tout». Mais l' "un» ne finit pas par s'identifier au «tout» tandis que «tout» tend toujours vers l'un. La totalité tend, elle-aussi, à se résorber dans l'unité tandis que l'unité, dans sa simplicité, demeure sans attrait pour l'ensemble composé dont la totalité doit s'abstraire pour exister ${ }^{16}$. Il s'agit, au fond, de l'asymétrie fondatrice et foncièrement dialectique du rapport entre l'un et le multiple, qui s'étend nécessairement sur les définitions du «tout» et de la «totalité» ${ }^{17}$.

Qu'il soit question d'une pluralité d'individus dont la réduction passe par l'universel prédiqué de leur genre, ou que les parties elles-mêmes se muent afin d'assurer la perfection du corps qu'elles composent et qui devient à terme l'unité au-delà du composé, ces identités relèvent d'une essence commune qui transcende aussi bien le multiple que l'unité en soi de chaque partie composante. Cette essence commune est déterminante, et les parties d'un «tout» ou les composantes d'un ensemble ne peuvent pas, par conséquent, constituer une altérité véritable à l'égard de l'entité du "tout» sur le fond de leur différence transitoire. Le «tout» est à la fois l'ensemble et cette essence qui dépasse l'ensemble et qui se trouve inscrite comme identité transcendante dans chaque composant, car, dit Aristote dans le

15. Je reprends cette expression que j'ai utilisée pour désigner le statut théologique de l'image dans Eikôn. L'image dans le discours des trois Cappadociens, Paris, PUF, 2010.

16. Dans le contexte spécifique de la philosophie aristotélicienne, une analyse de ces questions imposerait de mettre en parallèle les chap. 26 et 6 du livre Delta, et en particulier la partie consacrée dans le chap. 6 à la définition de l'un par essence (1015b 36 et sq.): voir, par exemple, le passage sur les êtres dont le genre est un (1016a 23-30) ou celui sur la définition de l'un indivisible comme principe pour le nombre (1016b 17-20). Nous n'entamons pas cette analyse approfondie, car elle nous éloignerait du but proposé dans cette étude.

17. Rappelons que Platon attribuait à la "gent Eléatique ( ce rapport entre l'unité et le tout ( $\dot{\varepsilon} v o ̀ \varsigma ~ o ̋ v \tau o \varsigma ~ \tau \tilde{\omega} v \pi \alpha \dot{\alpha} v \tau \omega v \kappa a \lambda o v \mu \varepsilon \dot{\varepsilon} v \omega v$ ) qui est alternatif à la relation entre l'un et le multiple. Il dénonçait la poursuite de cette question dans les mythes, là encore par une approche indirecte (Sophiste, 242d 5-7 et 242e 1-243a 2). 
prolongement du chapitre consacré au holon, «il faut aussi que l'essence

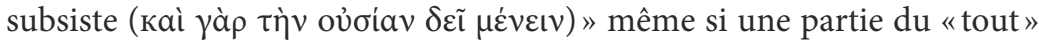
venait à être retirée ou mutilée ${ }^{18}$. Essentiellement, une coupe tronquée est encore une coupe, cite Aristote en guise d'exemple (1024a 16-17). Dans le cas d'un ensemble de cette nature, le «tout» demeure en tant que "tout» même s'il est modifié, et son essence est présente dans toutes les parties sans pour autant les modifier en tant que parties. Or, l'expression de cette réalité essentielle de l'ensemble qui transcende la somme des parties d'un tout-entier est désignée comme «totalité».

En somme, Aristote établit deux sortes d'unité du «tout»: soit «ensemble» de parties qui sont des unités en soi (de monades ou des individus), soit «tout» à partir d'un ensemble de parties qui ne constituent pas des unités indépendantes en soi (les membres d'un corps, par exemple). Et pour chacune de ces catégories, le Stagirite donne comme exemples privilégiés l'ensemble des vivants (ä $\pi \alpha v \tau \alpha \zeta \tilde{\omega} \alpha, 1023 \mathrm{~b} 33$ ) pour la première,

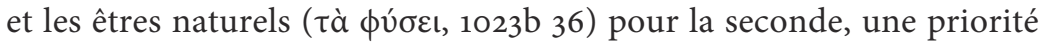
axiologique assignée donc aux vivants dans l'acte par lequel la totalité s'accomplit comme unité. Par la suite, le philosophe prend l'exemple des nombres pour distinguer un ensemble morcelable de parties semblables ou dissemblables, d'un ensemble d'unités et d'un tout naturel continu, tel l'eau ou le feu.

Sans s'attarder davantage sur les difficultés soulevées par le texte aristotélicien, on peut d'ores et déjà retenir, outre le lien indéfectible entre «tout» et «un », que la manière de définir le rapport du «tout» envers les parties et des parties envers le «tout» est présentée comme asymétrique dans les deux textes et que la différence responsable de cette asymétrie provient dans les deux cas du statut des parties elles-mêmes, même si pour l'auteur du Liber ce statut se détermine en fonction de l'accès au "tout» et non selon la nature, autonome ou dépendante, des parties elles-mêmes à l'égard de l'ensemble qui les comprend. A contrario, il faudrait également retenir que cette approche des parties est absente de la discussion concernant l'un et les parties selon la première hypothèse du Parménide $(137 \mathrm{c}-\mathrm{d})$, les parties étant évacuées au nom de la pluralité qui s'oppose à l'unité de l'un. Si secundum formam semble indiquer le Parménide comme source d'inspiration, la définition IX et sa glose le démentent puisqu'elles traitent le rapport un-tout d'une manière différente de celle présentée dans le dialogue. De même, entre le passage de Delta et le commentaire

18. Métaphysique, Delta, 27, 1024a 15. Voir au sujet du lien entre le chapitre sur le «Tout» et le chapitre sur le «Tronqué», le commentaire de R. Bodeüs et A. Stevens (in Aristote, Métaphysique, Delta..., p.197). 
de la définition du Liber, une concordance semblable se manifeste aussi au sujet de la totalité, tandis que cette notion est absente dans le Parménide qui se résume à discuter l'opposition holon/meros. On désigne par «totalité» (holotês ou totalitas dans les deux textes ici cités), le propre de l'unité (en l'occurrence, la deitas du Dieu un et seul dans le cas du Livre latin), sans toutefois identifier totalité et unité, car la totalité n'est qu'une sorte d'unité, est comme une expression ou une manifestation de celle-ci, de même que l'unité elle-même est le propre de l'un et le sous-tend, sans constituer pour autant son identité. Avec les mots latins: deitas n'est pas Deus, et totalitas livre le propre de la deitas mais n'est pas le nom propre de celle-ci; semblablement en grec: holotêtos n'est pas henotêtos, chacun ayant son propre nom.

Cependant, ce parallélisme avec le livre de la Métaphysique cache aussi un écart radical, tout aussi significatif que les possibles emprunts ou échos signalés. Dans le schéma aristotélicien le tout et les parties sont saisis comme appartenant au seul plan de réalité des étants, la discussion se focalisant sur la possibilité de distinguer entre l'unité des parties indivisibles tenant ensemble par leur appartenance en tant qu'individus à un ensemble avec lequel ils partagent le trait commun d'un genre, et l'unité de parties qui n'ont pas la possibilité d'exister autrement que dans l'ensemble dans lequel elles se trouvent en puissance ou en acte (tels les facultés et les organes d'un corps qui sont des parties mais non des individus d'un ensemble). L'analyse d'Aristote porte sur le seul plan de la réalité immanente, mais, de toute évidence, le plan sur lequel se tissent les deux liens entre tout et parties dans la définition IX ne peut pas être le même, car l'auteur du Livre latin le réduit à un cas sans genre, le cas unique de Dieu. Si un dieu figure aussi chez Aristote parmi les trois vivants cités, celui-ci est un participant du multiple puisqu'il est compris sous le genre divin, alors que dans le cas du Deus latin, Dieu est seul et unique en son genre, son unicité n'étant pas celle d'une unité arithmétique. Il s'ensuit que l'asymétrie du rapport entre le tout et les parties ne peut pas être considérée de la même manière. Pour le Stagirite, elle est structurelle au plan ontologique décrit, tandis que le commentaire de la définition du Liber, qui semble partager la description méréologique aristotélicienne, stipule, elle, une asymétrie cognitive et donc une sorte de prééminence de l'un sur le tout inhérente à cette démarche. Notons cependant que cette asymétrie cognitive stipulée dans le commentaire de la définition IX du Liber n'apparaît pas comme déterminée secundum formam. Elle n'identifie pas, autrement dit, l'un et le tout à des «formes» (eidê), ni ne considère la séparation de l'un comme une opération propre à l'intellect. La différence avec l'approche aristotélicienne n'indique pas nécessairement une position parménidienne. Le «tout», dans la glose latine, réclame en effet une succession pour être saisi, 
alors que la nature divine est unique, simple, indivisible et séparée - qu'elle soit envisagée à partir de sa totalité (ici: au-delà de la succession que suppose l'existence relevant du multiple), ou qu'elle soit appréhendée à partir de chacune des parties dans laquelle Dieu est saisi comme ce qui simultanément la détermine et la transcende, à l'instar de l'instant «présent», déterminant mais séparé de la succession immanente à la temporalité.

Autrement dit, l'auteur du Liber engrange les éléments de la leçon aristotélicienne, y compris l'idée d'un dieu vivant parmi les vivants sous le mode du Dieu présent (praesens), mais dispose la démonstration de telle manière qu'une différence absolue puisse remplacer la différence transitive installée par la démarche métaphysique aristotélicienne entre la nature et l'identité du tout et de l'un. Force est d'accepter toutefois qu'une contradiction accompagne dans ce cas la définition du Liber, car si Dieu n'est pas «un", comme chez Aristote, mais Monos (seul et unique), le propre de la déité ne peut pas être alors qualifié de «totalité des parties successives » sauf si totalitas désigne ici la transcendance de l'ensemble des parties, que celles-ci soient réunies en tant qu'entités autonomes dans un ensemble ou qu'elles soient des composantes dépendantes de l'unité. Si le propre de la déité est bien la totalité, alors totalité est ici synonyme d'unité, unité ayant le sens absolument simple et parfait de l'un en tant que principe d'unité. Ou alors, la déité n'est envisageable comme totalité qu'à une condition près, à savoir que la déité communique à la totalité la puissance de comprendre non pas les parties du «tout» une à une, successivement, mais simultanément le «tout», solution que semble proposer, sous l'appellation de «vue», la glose de la définition IX du Liber.

\section{Totalité et temps}

Dans la glose de la définition IX le rapport unité-totalité croise, explicitement, les questions de l'éternité divine et de la temporalité ou séquentialité de la perception. On peut penser que cette approche détournée par le temps, déterminée, certes, par la définition commentée (Deus est/ cui / soli praesens est quicquid cuius temporis est), permet de déplacer l'attention d'une trop évidente induction du multiple à partir de l'un. On éviterait ainsi le risque de faire de la définition du Dieu-monade un cas particulier des réalités incorporelles qui proviennent de l'un comme principe et qui, se déployant par la procession à travers laquelle le multiple est engendré à partir de l'un, retournent finalement à l'unité par un renversement du mouvement génératif ou productif ${ }^{19}$. Le caractère ramassé de l'assertion latine laisse en

19. Une définition de ce double processus des entités incorporelles, véritable résumé du topos néoplatonicien de la procession et du retour, est formulée par Porphyre dans la Sentence 11: 
effet place à de multiples conjectures. Mais le fait d'asseoir la question sur le plan temporel n'est en réalité pas sans rapport avec les difficultés que soulève précisément l'aspect partiel et successif qui caractérise l'approche «méréologique» de l'unité en regard avec une totalité comportant à la fois le tout et les parties. Là encore, le «tout» en tant que totalité tend à échapper à une approche fragmentée, propre au multiple même lors de sa «conversion" vers l'un, mais n'arrive pas à extraire la définition du Dieu un, seul et séparé bien qu'égal au tout-entier, de la partialité et donc de l'implacable imperfection de la composition et du multiple qui sous-tendent un ensemble même lorsque celui-ci est saisi par une vision globale.

Voyons maintenant de plus près le paradoxe temporel, celui qui semble arracher le présent au temps et le situer face à tout ce qui participe du temps. Exposé de manière extrêmement condensée dans la définition de la Monade divine, ce paradoxe s'éclaire dans toute sa complexité à travers la juxtaposition de quelques passages que Plotin et Porphyre ont consacrés à la perception du temps. Encore une fois, il ne s'agit pas d'identifier ainsi les sources textuelles du Livre des XXIV, mais de rendre davantage accessibles les définitions en reconstituant les champs philosophiques dont elles relèvent et les thématiques qui se croisent dans les formulations brèves de cet $o p u s^{20}$. L'usage du paradoxe temporel est éclairant en ce sens, puisqu'il met en relation trois notions: le tout, l'acte en tant que mouvement et l'ordre (ou la taxinomie des séquences ou des parties), notions auxquelles se réfèrent les occurrences textuelles des termes: éternité, moment présent et succession. De cette manière, l'asymétrie qui domine l'approche «méréologique» (la saisie de l'ensemble à partir de sa totalité ou à partir de chacune de ses parties une à une) peut être transposée au temps et donc assimilée à la structure perceptive (intellective et rationnelle) des unités temporelles (le «présent», le «maintenant»). Or ce déplacement est fondamental, car il permet d'écarter tout danger de projection d'une structure ontologique

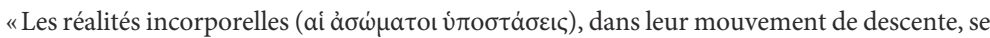
divisent et se pluralisent jusqu'au domaine de l'individuel par relâchement de puissance,

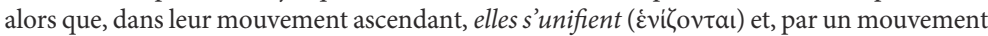

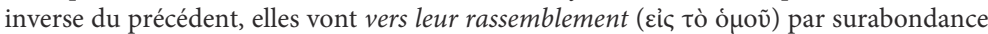

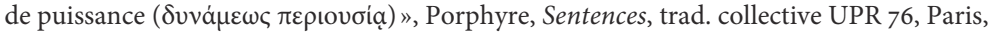

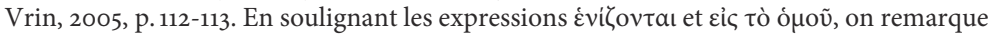
ce double mouvement qui traduit en termes néoplatoniciens l'asymétrie des rapports entre un, tout et les parties, asymétrie signalée dans l'analyse méréologique aristotélicienne de Delta 26 et que l'on pourrait aussi appeler en termes de donation-réduction puisqu'il s'agit ici du domaine de l'être, même si l'un est au-delà.

20. Certains de ces passages sont signalés par F. Hudry dans les notes qui accompagnent l'édition-traduction du Liber, d'autres ne le sont pas dans la mesure où le lien lexical ou thématique est moins évident. 
objective sur la définition de Dieu et de la déité, et enlève ainsi le soupçon de contradiction dénoncé plus haut, à savoir que la déité comporterait en tant que totalité une nature composite et une pluralité.

Certaines Sentences de Porphyre (en particulier 10 et 44 ) et quelques passages des Ennéades - en particulier III 7, 3 (Traité 45), mais aussi VI, 9 et 18 (Traité 39) - mettent plus particulièrement en évidence les grands axes du cadre conceptuel dans lequel s'inscrivent la définition IX du Liber et son commentaire. Certes, il s'agit d'un choix, non d'une liste exhaustive. En outre, ce choix est limité à des auteurs qui ont une notoriété certaine parmi les philosophes du IV ${ }^{\mathrm{e}}$ siècle, mais il est exclusif des traités théologiques de Victorinus dont les convergences avec les définitions du Livre des XXIV sont abondamment citées par Françoise Hudry, l'éditrice de ce dernier. Si rien ne prouve que l'auteur du Livre ait eu sous les yeux les traités de la Métaphysique ou les textes de Plotin et de Porphyre, faut-il pour autant s'interdire de voir dans les apories et les gloses du Liber aussi bien les échos des thèmes classiques de la réflexion, que les traces de l'argumentation philosophique utilisée dans les grands débats du premier siècle de la théologie spéculative chrétienne? Il est difficile de situer le Livre des XXIV définitions de Dieu dans le sillage immédiat du néoplatonisme gréco-latin du IV siècle sur la base d'une comparaison de textes issus d'œuvres à vocation et forme si éloignées du Liber que les Ennéades, les commentaires des Dialogues et des Traités ou même les Sentences. Tout comme il est surprenant de constater dans les choix théologiques du Liber l'absence de références explicites aux doctrines chrétiennes, ou du moins, la réduction de ces références à de subtiles et plutôt rares allusions trinitaires et christologiques décelables dans certaines définitions ou dans leurs commentaires (I, II, XIV, XXII), allusions qui paraissent suffisantes néanmoins, selon Hudry, pour considérer que «le texte présente une logique interne chrétienne ${ }^{21}$. Mais, au-delà de toute polémique historiographique et doctrinale, il n'est pas moins vrai que c'est à partir de cette juxtaposition de proximités et d'écarts textuels que l'on peut surprendre, en train de se tisser, les fils d'une certaine continuité entre les champs et les thèses d'une première pensée métaphysique et les théologies spécifiques néoplatoniciennes et chrétiennes, monadiques et structurées de manière triadique ou trinitaire.

Revenons aux rapports entre Dieu-un, tout, totalité et temps. Dieu un et seul du Liber est défini essentiellement comme celui pour qui est "présent» tout ce qui relève du temps. Serait-ce une définition analogue à celle de l'être,

21. Livre des XXIV..., p. 109 (voir p.101-111, ainsi que p. 25 pour une référence au Dieu monos d'inspiration philonienne, selon Hudry). Cette interprétation est longuement discutée par D. Coman, Ce este Dumnezeu?..., p.96-103. 
infinitif et indéterminé, face à la détermination inexorable de l'étant par le devenir? Nous ne pouvons pas aller dans cette direction, car Dieu monade est par définition au-delà de l'être. Puisqu'il est «le présent» de tout ce qui appartient au temps, Dieu ne s'identifie pas au temps, lequel se meut selon la succession, ni n'est dans le temps qui comporte des moments passés et futurs, à la fois multiples et changeants. Et puisque la déité correspond à la totalité du successif, la saisie exercée par celle-ci embrasse "tout» sans devoir passer d'une partie à l'autre selon l'ordre immanent de ce qui est fait ou produit, car, en embrassant «tout», la déité saisit simultanément «le tout» dans chaque partie. Le point nodal de ce processus est révélé par le terme praesens, le présent, l'incidence du maintenant qui se tient hors, et comme en face du temps. Pareillement, dans la glose, le regard unique saisit «tout» en se tenant hors de tout, et comme en face de l'ensemble pour pouvoir le saisir. Ce processus, dont on devine à peine l'esquisse dans les formules elliptiques du commentaire de la définition IX, est en revanche décrit en détail dans deux passages de la Sentence 44 (9-28 et 36-52) de Porphyre. Le premier passage de cette longue sentence concerne l'intellection simultanée de l'étendue et du temps par l'intellect qui est éternel, tout entier en tout (ö $\lambda$ ov ö $\lambda \omega$ ) et unique, agissant sous le mode de l'un dans l'un (ка $\theta^{\prime}$ $\tilde{\varepsilon} \nu \mathcal{\varepsilon} v \mathcal{\varepsilon} v \grave{i})$, tandis que le second passage décrit le mouvement de l'âme par lequel le temps subsiste, mouvement qui est circulaire, qui fait que l'âme se retourne sur elle-même quand elle connaît, et qui impose en même temps le parcours et la multiplicité des moments présents de la saisie ${ }^{22}$. Or cette description, de souche plotinienne d'ailleurs, permet de comprendre non seulement quelques emprunts qui paraissent incontestables dans le Livre des définitions ${ }^{23}$, mais aussi les points où l'auteur du Liber, de toute évidence un familier de la pensée de Porphyre et de Plotin, se sépare néanmoins de certaines positions défendues par les philosophes (néo)platoniciens.

Analysons la première description, celle qui concerne l'intellection, acte de l'intellect, et l'objet propre de cet acte, l'intelligible, en commençant par souligner que la manière descriptive de Porphyre pourrait signaler une sorte de définition secundum formam sans renvoyer nécessairement à l'archétype platonico-parménidien. Cette remarque n'est pas anodine.

22. Voir infra les passages évoqués de la Sentence 44.

23. Les proximités avec cette Sentence porphyrienne sont signalées aussi par F. Hudry qui cite brièvement les passages (Livre des XXIV..., p. 168, notes 3 et 5). Pour ma part, outre l'intérêt pour certains détails de ces proximités, j'insisterai aussi sur quelques distinctions porphyriennes qui ne se retrouvent pas dans le commentaire de la définition IX du Liber et dont l'identification pourrait éclairer des options philosophiques particulières de l'auteur latin. Mais l'énigme de l'identité de ce dernier et de la datation de l'opus demeure malgré tout entière. 
Elle signifie qu'il faudrait voir dans la modalité descriptive un rappel de la leçon presque méthodologique formulée par Porphyre dans la Sentence 10 qui stipule l'adéquation nécessaire de l'approche à l'objet approché, partant de la formule que "tout est en tout» ( $\pi \dot{\alpha} v \tau \alpha \mu \dot{\varepsilon} v \dot{\varepsilon} v \pi \tilde{\alpha} \sigma ı)^{24}$ et que c'est la visée et le mode propre de ce / celui qui envisage le «tout» qui assurent

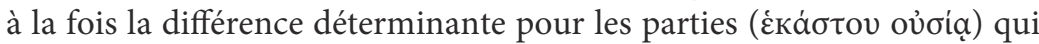
composent le «tout» en tant qu'ensemble, et la nature du «tout» lui-même. Il va de soi que cette formule «toutes les choses sont en toutes ( $\pi \dot{\alpha} v \tau \alpha \mu \grave{\varepsilon} v$ $\dot{\varepsilon} v \pi \tilde{\alpha} \sigma(v)$ » indique une approche parfaitement distincte de la similitude homothétique qui gouverne la théorie classique des formes. Par conséquent, la description de l'action propre à l'intellect donne une sorte d'image qui correspond à la nature du noûs à partir des noêta et de l'acte de la noêsis, sans révéler pour autant ce qu'est le noûs dans son entité entière et éternelle puisque cette approche essentielle est impossible en dehors de la description du mode de l'intellection (noerôs). On lit dans la Sentence 44 (9-14; 19-28):

Si donc l'intellect est intelligible et non pas sensible, il doit être objet intelligible; si d'autre part il est intelligible pour l'intellect et non pour les sens, il doit être sujet intelligeant. C'est bien le même (aủiòc) qui intellige et qui est objet intelligé tout entier par lui-même tout entier (ö̉ov ö $\lambda \omega$ ) [...] car il est sans parties, intelligible tout entier pour lui-même tout entier (ö $\lambda$ ov ö $\lambda \omega$ ) et intellect aussi en son entier (voṽ $\delta \mathrm{I}^{\prime}$ ö $\lambda \mathrm{ov}$ ), ne se pouvant même supposer qu'il ait en soi-même de l'inintelligence. [...] Mais si pour lui ceci ne vient pas après cela, c'est qu'il intellige toutes choses en même temps; puis donc que c'est toutes choses en même temps, et non pas l'une maintenant et l'autre ensuite, c'est toutes choses en même temps maintenant et toujours ( $\pi \dot{d} v \tau \alpha$ ă $\mu \alpha$ vข̃v кaì ảzí). Si donc pour lui il y a le «maintenant», et qu'aient disparu pour lui le passé et l'avenir, le voici dans une condition inétendue du fait de ce

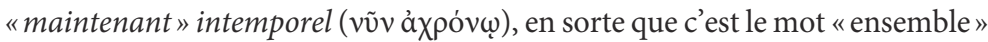
( $\tau o ̀$ ónoṽ) à la fois sous de la pluralité et sous l'angle de l'étendue temporelle,

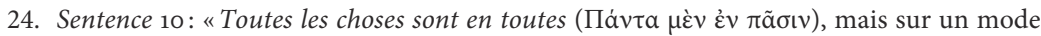

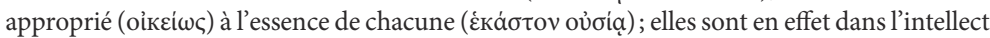

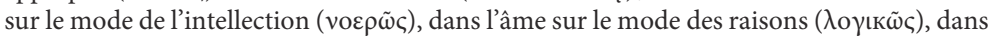
les plantes sur le mode de la semence ( $\sigma \pi \varepsilon \rho \mu \alpha \tau i \kappa \tilde{\omega} \varsigma)$, dans les corps sur le mode de l'image

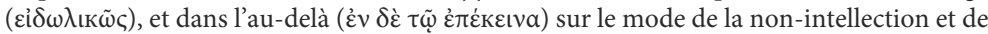

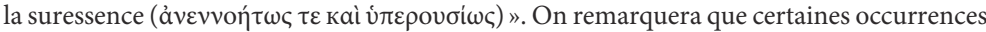
lexicales du «tout» (totus, totum, tota) du Livre des XXIV sont associées à des définitions de Dieu selon les modes et les domaines spécifiques signalés par Porphyre. Voir, par exemple, les définitions VIII (sperma quo vita tota), XVI (Dieu secundum se totum selon le mode par lequel le langage signifie ce qu'intellige la mens, ou selon le mode exemplaire de l'espèce qui se trouve ou non dans l'âme) ou encore les définitions II et XVIII (sur le mode imagé, sphérique, d'envisager modum imaginandi le «tout» sans dimensions de Dieu). Mais force est de remarquer qu'il s'agit plutôt d'échos que d'une application systématique de la «leçon» porphyrienne dans la théologie esquissée par les définitions latines commentées. 
qui s'applique à lui; aussi intellige-t-il toutes choses sous le mode de l'unité (ка $\theta^{\prime}$ ' $\left.\tilde{\varepsilon} \nu \pi \alpha \dot{v} \tau \alpha\right)$ dans une unité à la fois inétendue et intemporelle. Mais si c'est cela, il n'y a pas dans l'intellect le passage d'un point à un autre, ni par

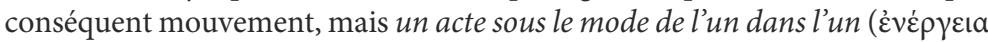

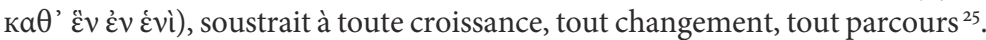

S'il est évident que l'intellect correspond ici au Dieu de la définition IX (et implicitement à la deitas du commentaire), Dieu dont la capacité de saisie du «tout» abolit l'étendue et la pluralité des parties de l'ensemble, et dont l'éternité se lit dans le caractère atemporel de son "présent» à lui

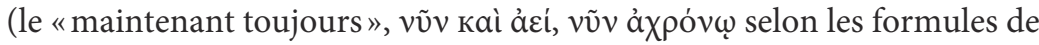
Porphyre), la saisie unique à partir du «tout» étant quasi identique avec la description de l'intellection, en revanche, le principe qui détermine les conditions spécifiques de l'intellection n'est pas le même dans le cas de la vision synoptique qui caractérise le point de vue de Dieu sur le déploiement du multiple à partir du «tout ». L'intellect ne peut que se connaître lui-même, car rien de ce qui n'est pas intelligible n'est en mesure d'être appréhendé par l'intellection. L'intellect connaît donc ce qui n'a aucune dimension sensible et n'existe pas dans le temps mais dans un présent perpétuel, à l'image de l'éternité du noûs, et connaît ainsi du «tout» l'unité parfaite de l'un (comme «un dans l'un») puisque l'unité aussi est à l'image de sa nature. En somme, l'intemporalité du simultané et l'inétendue, de même que l'unité et l'homogénéité de l'ensemble face à la pluralité, caractérisent le mode par lequel l'intellect saisit en «tout» ce qui de facto est en condition d'identité avec lui-même et lui permet ainsi de s'identifier sans amoindrir sa perfection grâce à cette identité entre l'acte, l'actant et l'objet sur lequel porte l'acte. Il y a certes des similitudes frappantes dans cette description avec la définition secundum formam du Dieu monade du Liber. Cependant, si on cherchait la notion de totalité dans ce contexte, il faudrait se résoudre à constater qu'elle n'est pas présente de la même façon que dans le commentaire formulé par l'auteur latin, voire qu'elle est en réalité absente.

Certes, il est question de «tout» comme unité et d'ensemble homogène qui transcende la pluralité. Mais totalitas, nous l'avons vu, n'est pas exactement cela. Porphyre utilise d'ailleurs un mode de désigner le tout du «tout» qui ne trompe pas sur la signification assignée. Il utilise, et même deux fois, le dédoublement du même terme par déclinaison: ö $\lambda o v$ ö $\lambda \omega$. Sans que l'on puisse voir dans ce dédoublement un usage adapté de la figure rhétorique de l' «épanaphore » qui donnerait à la relation du même au même que l'intellect entretient avec lui-même le sens fort d'une autocausalité

25. Porphyre, Sentences..., trad. légèrement modifiée, p. 374-375; c'est moi qui souligne. 
réflexive ${ }^{26}$, force est d'admettre que «tout » signifie ici l'entité complète et entière d'un ensemble qui demeure immanent à lui-même, sans indiquer en même temps l'unicité et/ou l'universalité de cette entité. «Tout» n'a pas pour vocation propre la totalité mais reste toujours «tout», enfermant dans cette condition la promesse d'une pluralité de «touts» (comme des classes de choses ou de réalités incorporelles ou d'intelligibles). La totalité en revanche, dont le nom ne répète pas à l'identique le même mot que le nom du «tout» bien qu'il soit issu de la même racine (comme totalitas de totus ou holotês de holos), semble esquisser dans la glose latine que la saisie, dont la totalité fait l'objet en même temps qu'elle indique la nature de l'agent, n'est pas une saisie de l'identité avec soi mais précisément de ce qui n'est pas soi. La saisie opérée par le biais de la totalité cherche à atteindre ce qui étant différent, étant autre que soi, comme la succession propre à la pluralité, peut être converti à partir de chaque partie et ramené à l'unité depuis les parties, en réalisant ainsi que ni l'unité ni la totalité (qui est une sorte d'unité) ne s'identifie avec l'ensemble réuni des parties composantes. En raison de quoi, la totalité signifie en tant qu'unité la transcendance du «tout» et, se distinguant de l'universel dans le cas de Dieu qui ne connaît pas le genre, elle indique aussi que la transcendance du «tout » est de facto universelle et rejoint le principe du «tout», sauf dans le cas unique et seul de Dieu qui n'est pas principe mais donateur du principe. On comprend mieux l'écart par rapport à la description de Porphyre en remplaçant totalitas par deitas: la déité est pourvue d'une vision unique qui saisit l'unité dans la pluralité et à travers la succession des parties, mais cette unité à partir du «tout» n'est pas l'image de la déité, et encore moins une image de Dieu. L'unité à partir du «tout» et de chaque partie indique, en revanche, la manière de transcender le «tout » et constitue en même temps la garantie de la différence absolue par rapport à la pluralité, à la diversité et à la partialité d'une saisie immanente.

La seconde description de la même Sentence porphyrienne déploie les conditions du rapport de la connaissance au temps en déplaçant le sujet depuis l'intellect vers l'âme. Dans la Sentence 44 (36-52), on lit:

C'est l'âme qui passe d'une chose à l'autre, échangeant pour d'autres les produits de son intellection, non que les premiers s'écartent ni que les autres s'introduisent de quelque côté, mais les uns semblent avoir passé bien qu'ils demeurent en elle, les autres leur succèdent comme s'ils venaient d'ailleurs, alors qu'ils sont arrivés non pas d'ailleurs, mais de l'âme quand elle se meut

26. F. Hudry propose ce rapprochement mais au sujet de la définition XVII, où l'allusion semble en effet transparente (Livre des XXIV..., p.186, note 3). 


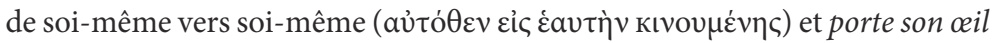

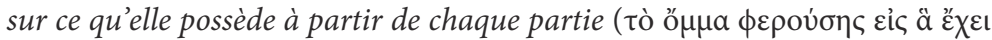
ката̀ $\mu \varepsilon \dot{\rho} \rho \varsigma)$; car elle ressemble à une source qui ne se déverse pas, mais fait refluer circulairement vers soi-même ce qu'elle possède. Ainsi donc, c'est en dépendance du mouvement de l'âme que subsiste le temps, en dépendance de la permanence en soi-même de l'intellect que subsiste l'éternité, laquelle n'est pas séparée de lui plus que le temps ne l'est de l'âme, puisque même les réalités dont l'existence est dépendante sont unies là-haut. Mais ce qui est en mouvement, <face à ce qui est en repos $>$ usurpe une éternité qui lui

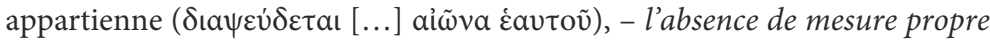

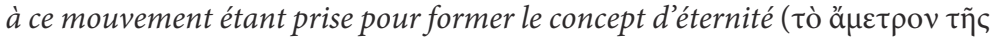

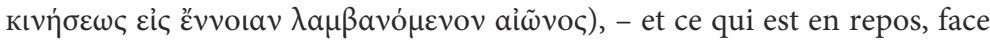

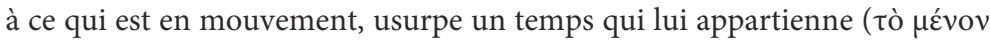

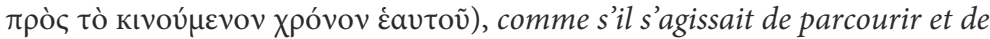

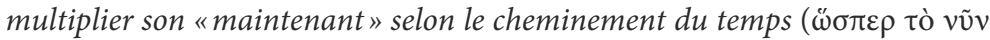

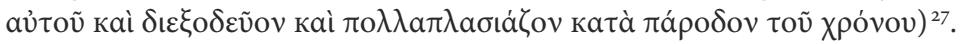

Nous trouvons ici, dans la suite immédiate du précédent passage, le vocabulaire qui manquait pour compléter le tableau des correspondances avec le commentaire de la définition IX du Liber: l'œil qui embrasse tout et la vision qui distingue chaque partie; l'articulation conditionnelle entre temps et mouvement, éternité et permanence, présent et multiplicité enchaînée de «maintenant(s)»; enfin, le mouvement par lequel la faculté d'appréhension parcourt l'étendue en cheminant d'une chose à l'autre. Mais, à travers cette thématique commune se manifeste aussi la divergence de positions entre Porphyre et l'auteur anonyme du Liber. Par rapport à l'intellect dont l'exercice consiste à s'identifier essentiellement lui-même, l'âme ne fait que se retourner vers elle-même dans un mouvement circulaire de repli qui succède à une sorte d'épanchement contenu entre des limites idoines à faire refluer vers la source tout ce qui se déploie en même temps à travers son mouvement continu. Ce mouvement simultané d'épanchement (de "sourcement» pour ainsi dire) et de réflexivité de l'âme a deux effets. L'un consiste à faire de l'âme le relais du temps. Plus précisément, le mouvement circulaire de l'âme donne possibilité d'existence au temps et en assure la forme sous le mode d'un renouvellement cyclique reprenant le trait du reflux circulaire de l'âme, tandis que la subsistance permanente et identique de l'intellect auprès de lui-même donne et se porte garant de l'éternité. À cet effet temporel du mouvement de l'âme collaborent le mouvement lui-même et l'absence ou la présence d'une mesure qui tantôt fausse le mouvement (par son absence, ametron) en laissant ainsi la place au repos et à l'éternité,

27. Porphyre, Sentences..., p. 376-377, trad. légèrement modifiée; c'est moi qui souligne. 
et tantôt contrarie le repos en laissant s'enchaîner de multiples moments présents, en multipliant, dit Porphyre, les «maintenant(s)» (nun), jusqu'à en faire le cheminement propre du temps. Cette multiplication des moments présents semble renvoyer au "présent» auquel s'identifie Dieu unique et seul face à ce qui est dans le temps. Mais la ressemblance est dans son fond mensongère, car pour Porphyre l'enchaînement de moments présents est censé donner le temps en y participant, tandis que dans la définition du Liber le présent dans lequel Dieu se tient en retrait par rapport à ce qui est (dans) le temps (praesens quicquid cuius temporis est) constitue, au contraire, une mise à l'écart et une manière de signifier la perpétuité du présent dans lequel Dieu se laisse penser, appréhender par opposition au temps. De même, la vision simultanée de toutes les parties signifie la possibilité de la connaissance selon l'étendue, mais nécessairement en opposition avec la multiplicité et le cheminement pas à pas à travers celle-ci. C'est comme si l'auteur du Liber avait voulu prendre à contre-pied le raisonnement porphyrien en en faisant une lecture subtile et en le renversant à partir de ses propres mots et arguments, sur le terrain proprement philosophique et non sur celui d'un débat doctrinal ${ }^{28}$.

Le second effet du mouvement circulaire de l'âme tient de l'exercice perceptif qui s'actualise ainsi. L'âme voit, selon Porphyre; elle voit tout et se voit en tout. On peut se rappeler un passage de Némésius qui assignait à Porphyre cette théorie de la vision de l'âme ${ }^{29}$, en l'exprimant plus explicitement encore que le texte de la Sentence 44. Le mouvement d'extériorisation de l'âme, mouvement comparé au jaillissement d'une source, nourrit en effet comme une source, en continu, tout ce que l'âme perçoit en revenant par retour réflexif sur elle-même. Elle donne et en même temps perçoit ce qu'elle donne en se retournant vers elle; elle voit ainsi le multiple qui provient de

28. Notons au passant que ce décalage entre la sentence porphyrienne et la glose latine pourrait éclairer les lignes de fracture et les raisons d'un autre décalage qui se fait jour dans ce contexte: l'éloignement, subtil mais net, entre l'usage des genres dits «de l'être», comme le mouvement et le repos, rappelant les dialogues de Platon, et le développement spéculatif de Porphyre qui ne suit pas ici l'argumentation du Parménide ou du Sophiste.

29. Némésius d'Emèse, De natura hominis VII: « Porphyre dit, dans son traité de la Sensation,

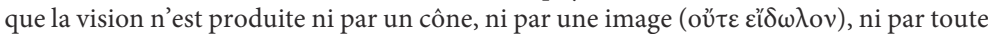
autre chose, mais que l'âme, mise en rapport avec les objets visibles, ne fait que se voir [se

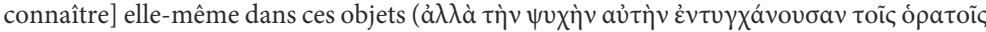

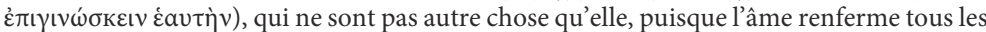
étants et que tout ce qui n'est rien d'autre que l'âme contenant les corps de toute espèce

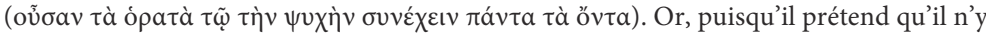

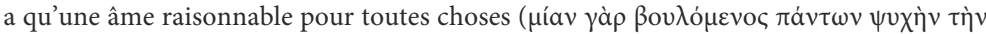

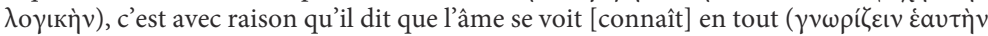

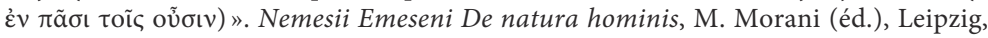
Teubner, 1987. 
son propre sourcement intarissable, mais le perçoit dans l'unité à laquelle le multiple se réduit de lui-même en retournant à l'âme. Cette unité est traduite par l'unité temporelle du «maintenant» qui signifie l'actualisation de l'unité de l'âme avec elle-même, unité qui est aussi la puissance contenue de l'âme, représentée par la métaphore de la source. Il s'agit en réalité du même mouvement double de donation-réduction, traduisant la simultanéité dans l'âme de la puissance et de l'acte, mouvement que Porphyre décrit dans la Sentence 11 en l'attribuant aux réalités incorporelles ${ }^{30}$. Il s'ensuit, cependant, que ce mouvement ne peut qu'exclure la possibilité d'envisager une totalité à l'égard du "tout» en même temps que distincte de celui-ci, si ce «tout» est à la fois donné et réduit à l'unité. Pourquoi ce mouvement de flux et reflux exclut-il cette possibilité? Parce que l'âme, même si elle n'est pas dans l'identité permanente avec elle-même comme l'intellect, n'a aucune possibilité de connaître quelque chose qui ne provient pas d'elle et ne retourne pas à elle. En donnant "tout» dans son déploiement, elle réduit en même temps «tout » à l'unité avec elle. «Tout» comprend donc "tout», y compris la totalité sous l'espèce de l'unité ${ }^{31}$.

Or la totalité, telle qu'elle apparaît dans le Livre des définitions, de même que dans la définition aristotélicienne, est irréductible au «tout»; et si elle constitue une "unité», celle-ci ne s'identifie pas à la somme de ce qui compose le «tout» mais à ce que comporte comme indice d'appartenance $\mathrm{au}$ «tout» chaque partie d'un ensemble. C'est donc la possibilité d'une saisie de l' «entier» au-delà du «tout» dans chaque partie qui constitue la garantie de la totalité, et qui conditionne en même temps sa distinction du «tout», qui assure même la transcendance de la totalité à l'égard du " tout», sa parenté avec la notion d'universel dans le cas des classes d'entités déterminées elles-mêmes comme des unités selon la définition du Stagirite, ou bien son statut d'expression de la puissance qui engendre la connaissance propre exercée par la Monade divine, telle qu'elle est définie dans le commentaire de la IX ${ }^{\mathrm{e}}$ définition aporétique de l'opus. Si le syntagme deitas successivorum totalitas fait suite à la différence de vue à partir du tout et à partir de la partie, il faut alors comprendre que le propre de la déité, y compris sa puissance de connaître le tout, s'actualise en effet dans la totalité, au-delà du successif et donc du multiple des entités individuelles saisies comme des parties. Dès lors, la totalité doit être comprise comme contraire à la fois à l'étendue du multiple et au déploiement temporel

30. Texte cité supra note 19.

31. On revient ainsi à la formule « tout est en tout» de la Sentence 10 et à la nécessité d'établir une distinction modale pour faire place à la différenciation au sein de cette unité parfaitement solidaire de son identité. 
dans lequel s'effectue la saisie. Autrement dit, elle, la déité, comporte la possibilité de s'identifier à la totalité à travers la succession temporelle et à travers l'étendue, de même qu'à travers la diversité du multiple, puisque ni la totalité ni a fortiori la déité ne participe de la succession, de l'étendue et de la pluralité auxquelles intuitus unicus, le regard unique (de Dieu?) a pourtant accès.

Ne participant ni de la temporalité (nécessairement successive), ni de l'étendue, ni de la pluralité des parties ou des entités individuelles, la totalité correspond parfaitement à la nature de ce regard unique et seul qui, ouvert sur le successif, le pluriel, l'étendu et le divers, saisit dans la perpétuité de l'instant la totalité manifestée dans chaque partie du successif, du pluriel, de l'étendu et du divers. Ce coup d'œil unique saisit donc ce qui correspond à sa nature dans ce qui n'est pas lui. Mais comment comprendre qu'il peut saisir dans ce qui est différent ce qui lui correspond? Qu'il voit dans ce qui se voit ce qui n'est pas visible à un regard cheminant d'une chose à l'autre comme s'il lisait un livre? La possibilité de comprendre ce défi se cache peut-être dans le choix, tout sauf anodin, du lexique de la vision. N'est-il pas remarquable que le seul acte nommé dans la glose de la définition IX, hormis l'être prédiqué de Dieu et du temps, est précisément l'acte visuel, video, acte qu'exerce le tout, totum... videt? Et que cet acte est lui-même défini par la possibilité d'un recours double, comme si son exercice était différent selon deux points de vue, l'un de prééminence, l'autre de découverte de l'identité, bien que leur point focal soit le même.

Le recours à Plotin pourrait s'avérer salutaire pour mieux éclairer les questions que soulèvent le rapport intellectif au temps et à l'éternité, l'exercice spécifique d'ordre visuel de la pensée et le rapport de cette "vue» avec la relation unité-pluralité des intelligibles, relation sous-jacente à la recherche d'un accès au tout ou à la totalité par l'intermédiaire de l'unité dans laquelle réside l'identité de chaque chose avec elle-même. Mais, comme dans le cas d'Aristote ou de Porphyre, les quelques fragments des Ennéades que l'on peut évoquer ne sauraient couvrir l'ensemble de ces questions et sont nécessairement le résultat d'un choix. De même, comme à l'égard des Sentences de Porphyre, l'auteur du Livre latin des définitions se trouve dans une évidente proximité lexicale et thématique avec certains passages des traités plotiniens, mais ce vocabulaire et ces références thématiques servent à défendre par des écarts contextuels subtils une position philosophique différente de celle prônée par Plotin au sujet du principe de la Monade, de l'être et des conditions de leur saisie:

Quel est donc le caractère d'après lequel ce monde-là [le monde intelligible]

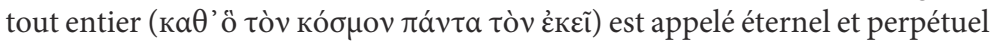




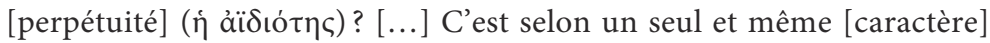

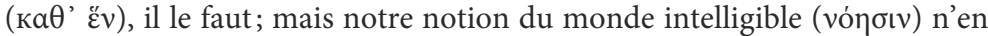
n'est pas moins faite de l'assemblage de choses multiples ( $\dot{\varepsilon} \kappa \pi \mathrm{o} \lambda \lambda \tilde{\omega} \nu)$. Certes, il y a une nature unique qui accompagne tous les êtres intelligibles, ou bien qui leur est unie, ou enfin qui apparaît en eux; certes, tous les êtres intelligibles

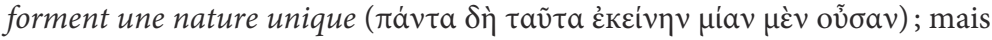
cette nature unique a des puissances multiples, et elle est plusieurs choses ( $\pi \mathrm{o} \lambda \lambda \grave{\alpha}$

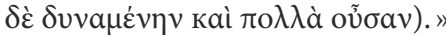

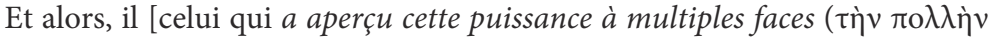

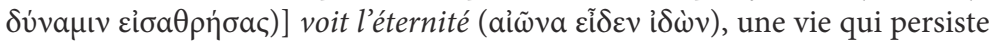
dans son identité ( $\dot{\varepsilon} v \tau \tilde{\omega}$ av̉ $\tau \tilde{\omega})$, qui est toujours présente à elle-même dans sa

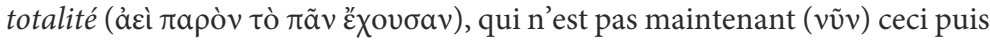
cela, mais qui est toute à la fois, qui n'est pas une chose puis une autre, mais

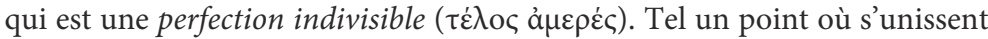
toutes les lignes, sans qu'elles s'épandent jamais au dehors; ce point persiste

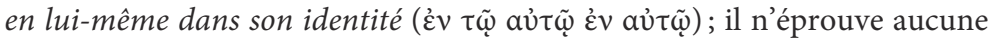

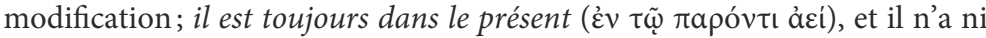

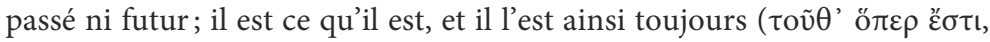

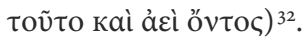

Il est certain que la proximité de certaines figures qui réalisent la synthèse de l'un et du multiple ou qui mettent en rapport éternité, temps et "présent

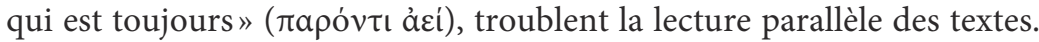
En particulier, elle soumet la définition IX et sa glose à une lecture induisant l'idée que la clé de ses formulations paradoxales se trouverait dans la métaphysique de type noétique esquissée par Plotin, dans cette pensée pour laquelle le cosmos se réduit à la relation d'unité entre l'intellect, l'intellection et ce qui s'y trouve à titre d'objet et de moyen de la pensée, à savoir les intelligibles (désignés comme "parties»). Porphyre, nous l'avons vu dans le premier passage cité du fragment de la Sentence 44, transpose cette unité en termes d'identité essentielle et structurelle (formelle) entre l'intellect et tout ce que l'intellect intellige, tandis que l'auteur latin semble traduire la relation d'unité comme une manifestation de la position prééminente qui revient à la Monade, Dieu un et seul jouant en tant que présent perpétuel le rôle de l'intellect en train de saisir, de "voir» la totalité dans l'identité de tout ce que déploie, comme étendue et succession, l'être dans le temps. Mais dans quelle relation ce Dieu un et seul se trouve-t-il par rapport à tout ce qui se déploie dans son étendue, sa pluralité et sa diversité ? Ce Dieu dont le propre, la deitas, correspond à la totalité, arrive-t-il à préserver

32. Plotin, Enn. III, 7 [Traité 45], 3, 1-7; 16-24. Plotin, Troisième Ennéade, E. Bréhier (trad.), Paris, Les Belles Lettres, 2002, p. 213, trad. légèrement modifiée et soulignée. 
sa perfection en se déployant ou est-il radicalement séparé de ce qui en provient? Plus précisément: ce «tout» qui «voit» l'ensemble des parties, est-il synonyme de Dieu parce qu'il est essentiellement identique à celui-ci comme à ce/ celui dont tout provient, ou bien Dieu un et seul se tient-il hors de tout ce qui procède de lui, préservant ainsi la structure absolue de son essence unique, parfaite et simple, essence à laquelle l'accès est en revanche assuré de manière séquentielle et partielle?

Secundum formam indique présentement cette seconde approche, mais elle ne la situe pas pour autant dans le sillage de la théorie des formes et des universaux. Si la totalité signifie que Dieu selon sa nature se trouve en dehors de la pluralité des parties et de leur succession, sa perfection étant indissociable de sa totalité et donc indivisible telle une entité infracassable, du point de vue formel, en revanche, tout ce qui en provient révèle cette totalité sous le mode successif et partiel, en la révélant de manière intacte à chaque moment du présent et dans chaque partie. Ce processus, qui comprend bien la totalité, ne peut cependant être saisi qu'à travers un cheminement. Le pas à pas ainsi suggéré, bien qu'il soit attribué au regard, concerne non la vue d'une image mais celle d'une forme discursive qui se développe par le déploiement de parties successives, inséparables et pourtant distinctes une à une comme les mots d'une phrase. Aussi le temps de ce qui est, passé ou futur, induit nécessairement une succession de formes verbales qui présentent, racontent ou décrivent de manière séquentielle un acte lequel, bien qu'unique et toujours effectif, telle la vie sans cesse en train d'engendrer la vie et de s'actualiser ainsi dans un présent perpétuel, ne peut être saisie qu'à travers des actions passées ou futures, jamais dans un «maintenant» absolu ou dans l'éternité indéterminée.

Pour Plotin, la synthèse de l'unité et de la pluralité se réalise dans et selon le modèle du noûs et des noêta. Plotin conçoit donc un modèle «méréologique» strictement noétique, visant à assurer les conditions de l'appréhension. En effet, l'un est séparé totalement. Seul le «témoignage» de son reflet dans l'intellect assure la possibilité de la pluralité des intelligibles d'une part, et de l'unité plurielle des puissances inscrite dans la nature unique des êtres de pensée d'autre part: « tous les êtres intelligibles

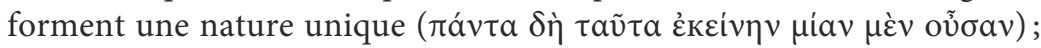
mais cette nature unique a des puissances multiples, et elle est plusieurs

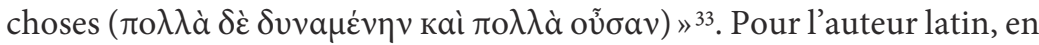
revanche, les identités mises en jeu par la relation «méréologique» ne sont pas les mêmes, et la ligne de fracture entre ce qui est dans le temps et ce

33. Plotin, Enn. III, 7 [Traité 45], 3, 6-7, fin du premier paragraphe cité supra. 
qui est indéterminé par rapport à l'être et au devenir, elle aussi ne suit pas le même trajet de la frontière entre le tout immanent et la transcendance de tout, transcendance dont l'expression est la totalité qui fait du tout tout et unité de surcroît. La raison en est que l'auteur latin introduit une relation d'asymétrie et que cette sorte de relation indique qu'à la différence de l'intellect qui ne connaît que ce qui tient de son identité et retourne toujours à celle-ci, Dieu ne connaît que ce qui en lui n'est pas lui et qui, dès lors, l'oblige à se pencher sur et vers lui comme du dehors. Dieu se tient dans l'équilibre fragile, bien que perpétuel, du présent, du «maintenant» qui se suffit à lui-même pour livrer une image entière de ce qui remplit le moment présent de son étantité. Équilibre fragile vu du dehors, car cela ne représente pas, bien évidemment, Dieu essentiellement, mais circonscrit le propre, la déité, la nature de ce qui fait de Dieu Dieu, comme la totalité par rapport à tout ou l'unité par rapport à l'un.

Secundum formam indique cependant quelque chose de plus sur de l'enjeu de la définition elle-même. Ce mode signifie aussi qu'une différence radicale doit être envisagée entre les notions de totalité et d'universel (kath'olou). Cette différence, qui sert en quelque sorte de garde-fou, définit ainsi la définition proprement dite, en signifiant le plan sur lequel se situe sa validité. Ce plan ne peut être que celui de la métaphysique, non celui de la logique; sur le plan métaphysique, la différence signifie dans ce cas la transcendance de la totalité par rapport à l'universel, tandis que sur le plan logique les deux paraissent équivalentes du point de vue formel. Non seulement la totalité transcende le tout et ne renvoie pas à l'identité qui enferme l'acte par réduction à l'objet dans le cas de l'intellect et de l'unitépluralité de nature des intelligibles, mais elle se distingue tout autant du statut des formes et ne dispose d'aucune universalité, puisqu'étant propre à Dieu (en l'occurrence, à la deitas) elle ne peut être définie ni selon l'essence ni selon les propriétés. Par conséquent, la définition, focalisée en effet sur la totalité, vise à mettre en évidence que celle-ci ne s'applique dans ce contexte qu'à l'unique et seul Dieu en tant que "présent", mais que cette application restrictive ne fait pas pour autant de la totalité une propriété de Dieu ou un nom divin ${ }^{34}$. Dès lors, mettre la définition sous le signe des formes indique en creux, comme par défaut, que la condition formelle ne s'applique, elle, qu'au rôle assigné aux parties et à la saisie du tout à partir

34. Voir dans ce sens la définition VI (sub relatione) du Liber qui interdit sous peine d'aliénation la circonscription de Dieu à une substance ou à une propriété commune ou accidentelle. Je déroge, en référant à cette définition, à la règle imposée à cette analyse qui consiste à ne pas appuyer une définition sur les acquis d'une autre, antérieure ou postérieure à celle étudiée, sauf lorsque la définition elle-même comporte une référence intertextuelle. 
de celles-ci, c'est-à-dire par la pluralité, l'étendue, la succession, lesquelles renvoient par retour du particulier à l'universel. C'est bien cette condition, en apparence fidèle à la première hypothèse de la théorie des formes (Parménide $137 \mathrm{c}-\mathrm{d}$ ) concernant le rôle des parties à l'égard du tout mais non à l'égard de l'un ${ }^{35}$, qui ne peut convenir à la totalité, alias à la déité, et donc $\mathrm{au}$ "point de vue» du tout, si celui-ci comporte simultanément la totalité et l'unité. Et dans ce dernier cas l'approche n'est pas formelle, ne fait pas appel aux universaux et n'est pas soumise aux règles de la définition discursive propre à la temporalité dans laquelle s'inscrivent les actions de tout ce qui est dans le temps. Plotin indique la vie, en guise d'exemple. Pour lui, la vie n'est pas soumise au temps, bien que tout vivant soit sous le coup de la métamorphose cyclique, car la vie est une perfection indivisible ( $\tau \dot{\varepsilon} \lambda$ oc ả $\mu \varepsilon \rho \varepsilon ́ \varsigma)$, propre à l'âme, et elle est par conséquent expression de l'éternité ${ }^{6}$. Mais l'auteur latin n'emprunte pas non plus cette voie. Sa stratégie est différente à la fois de celle de Plotin (visant l'éternité de l'âme, en l'occurrence) et de celle déployée dans la théorie classique des formes (visant par hypothèses l'absolue séparation de l'un).

L'anonyme latin introduit, pour sa part, une prééminence comprise sous l'image du regard qui embrasse tout et qui ne se soumet pas à la contrainte du parcours propre à une lecture discursive. Ainsi, sa définition secundum formam contient à la fois l'indication d'une définition selon la prédication (induite par la temporalité et suggérée par la succession des parties), et le dépassement de la prédication par une saisie globale, propre à la vision par laquelle Dieu connaît ce qui n'est pas lui en lui. On pourrait dire que la glose de la définition IX comprend ainsi à la fois l'indication de la division et le retour à l'unité, et qu'elle les comprend d'une façon "panoramique ", comme une synthèse dont le résultat n'est cependant pas déterminé par le dépassement de l'opposition un-multiple grâce à une tierce voie, mais par une transcendance de l'opposition elle-même à partir de ce qui n'est pas soumis à cette opposition, à savoir, la totalité précisément. La prééminence

35. Rappelons le passage bien connu: «S'il est un, n'est-il pas vrai que l'Un ne saurait être

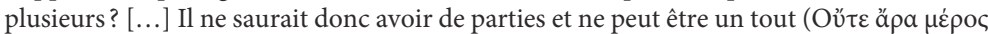

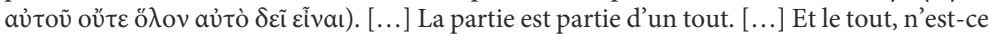
pas ce à quoi aucune partie ne manque? [...] Des deux façons donc l'Un serait composé

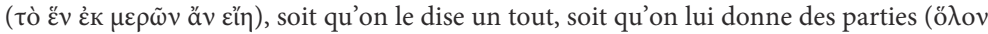
$\tau \varepsilon$ öv каі $\left.\mu \varepsilon \dot{\varepsilon} \eta \varepsilon^{\prime \prime} \chi 0 v\right)$. [...] Donc, de ces deux façons, l'Un serait plusieurs et non plus un

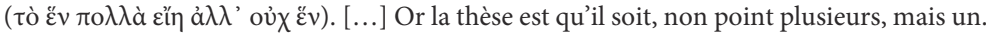
[...] Donc si l'Un doit être un, il ne sera point un tout et il n'aura point de parties (äpa

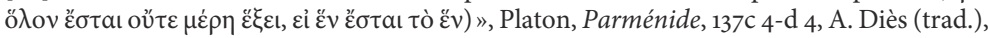
Paris, Les Belles Lettres, 1950, p. 72.

36. Plotin, Troisième Ennéade..., l. 16-17, p. 212-213. 
suggérée par la vision à partir du tout montre ce dernier aspect de la définition, aspect qu'il convient maintenant d'évoquer.

\section{Le coup d'œil : sunopsis et taxinomie}

Une autre définition du Liber est présentée non secundum formam mais comme formalis. Si le commentaire de la définition IX ne fait aucun renvoi à d'autres définitions commentées, et invite plutôt à une lecture autonome ${ }^{37}$, on peut justifier l'appel à la glose de la définition XI (haec definitio formalis est) en marge de l'analyse consacrée au commentaire de la définition IX, par la communauté modale entre les deux et par la nécessité de mieux comprendre ce qu'éclaire le choix déclaré du mode «formel» dans le contexte de cette série de définitions aporétiques de Dieu ${ }^{38}$. La définition XI aborde la question de la transcendance à l'égard de l'être dans le cas de Dieu ${ }^{39}$. Elle n'est d'ailleurs pas la seule à le faire, l'auteur revenant à plusieurs reprises sur le dépassement de l'être et de la substance (par exemple dans le commentaire de la définition XX), ainsi que sur la différence essentielle et la dissemblance entre Dieu, intellect et langage (commentaires des définitions XVI et XIX - cette dernière, en tandem avec la définition XX, formulant et glosant les figures aristotéliciennes du moteur immobile et de la pensée qui est acte par elle-même, vit en se pensant et est supérieure à l'essence, superessentialiter). Or, que dit au sujet de la forme la glose de

37. Ce n'est pas le cas avec d'autres gloses des définitions du Liber qui s'appuient entre elles, telle la glose de la définition $\mathrm{X}$ renvoyant explicitement aux commentaires des définitions IV et VII, ou la glose de la XVIII ${ }^{e}$ définition qui fait suite, sequitur, à la II ${ }^{e}$.

38. Notons que parmi les dernières définitions commentées de la série des XXIV, certaines ne présentent plus la structure indiquée plus haut (définition, mode, glose, parallèle). C'est l'indication modale qui disparaît dans les définitions XVII-XXIII, et dans plusieurs de ces cas un renvoi à une définition précédente prend la place de l'indication modale.

39. «DEUS EST SUPER ENS, NECESSE, SOLUS, SIBI ABUNDANTER, SUFFICIENTER. Haec definitio formalis est, et etiam a quo materia relata: "Esse omne dicit clausionem...". Superest igitur qui non clauditur. Est necesse quia malum non habet, quia non clauditur sed infinita possibilitate nec sic distrahitur suum et esse, cum redeat $\langle a\rangle$ se in se, et non totum indigenter, sed exabundanter». Soit «DIEU EST AU-DESSUS DE L'ÉTANT, NÉCESSAIRE, SEUL, À LUI-MÊME EN ABONDANCE, EN SUFFISANCE. Cette définition touche à la forme et se rapporte aussi au thème évoqué: "Tout être dit le caractère clos...". Il "est" donc sur le mode supérieur celui qui n'est pas clos. Il est nécessaire parce qu'il ne connaît pas le mal, parce qu'il n'est pas clos mais qu'il est d'une potentialité sans limite, et que son être même n'en est pas divisé, puisqu'il fait retour de soi sur soi, et non du tout par manque, mais par surabondance de puissance» (Livre des XXIV..., p.174-175). Hudry mentionne en outre que la référence avant la citation ferait allusion à la définition $\mathrm{X}$ et que la citation serait le rappel d'un passage du chap. 17 du livre Delta, 1022a 8-10 (ibid., p. 170, note 8). Notons, par ailleurs, que l'évocation du mal dans ce contexte mérite une analyse plus approfondie mais qui nous éloignerait du thème de cette enquête. 
la définition XI de Dieu? Elle affirme que la forme n'a pas de rapport avec le limité, car le limité ou le clos (clausionem) est propre à l'être (esse), or Dieu est supérieur à l'être. Qu'en outre elle ne connaît pas le mal; en clair, qu'il n'y a pas de possibilité d'opposition à l'être et qu'il n'y a pas non plus de forme possible du mal. Pourquoi? Parce que Dieu, en tant qu'illimité, possède la possibilité infinie, tandis que le mal, qui est manque ou privation d'être, n'a aucune possibilité hormis celle d'exercer une attraction conséquente à ce manque. Le mal réduit ou limite donc l'étant à s'identifier à sa finitude comme seule possibilité d'être et ne peut à ce titre avoir une forme (en d'autres termes, il n'y a pas de mal universel), tandis que Dieu, comprenant/ contenant l'être, ne connaît pas le manque et a, au contraire, la possibilité de décloisonner l'être à travers son propre dépassement. Ainsi, transcendant l'être, l'universalité de la forme donne en même temps à l'être la possibilité de s'autotranscender. Enfin, il est dit que la forme ne connaît pas la division, car Dieu, parfaitement simple, ne sépare jamais l'être de lui-même, bien qu'il ne soit jamais réductible à cette identité, et, s'il fait toujours retour sur soi, ce n'est pas par manque ou par nécessité d'identité avec soi, mais par surabondance, puisqu'en tant qu'illimité jamais il ne se quitte lui-même, qu'il s'abaisse ou qu'il se sépare en retournant à son état solitaire et suffisant. Nous comprenons que la division et le retour sont des mouvements qu'exercent les formes des étants, et qu'en raison de sa non-séparation Dieu suit lui-même ces actes, mais par surabondance non par manque, nécessité ou désir suscité par la privation. Françoise Hudry cite à cet égard la Sentence 11 où Porphyre décrit ce mouvement double en l'attribuant aux réalités incorporelles et en mentionnant que le retour,

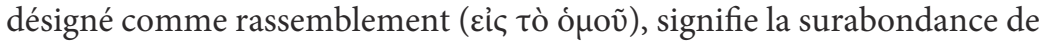

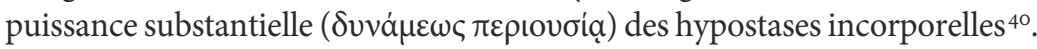

La proximité formelle de cette description cache cependant, une fois de plus, une différence de taille. La division et le rassemblement conviennent aux manifestations du composé ou aux natures parfaitement simples qui connaissent toutefois des opposés parce qu'elles relèvent de l'ordre des identités substantielles. Dieu, en revanche, n'est ni composé ni réductible (dans ce contexte) à une hypostase, à la réalité manifestée/actée d'un étant. Par conséquent, il ne retourne pas à soi mais il est toujours en soi - tel "un en l'un", selon le mouvement et l'acte évoqué par Porphyre dans la Sentence $44^{41}$. L'expression de sa puissance surabondante, puissance au-delà de l'acte, se révèle être elle-même acte par correspondance avec le statut de Dieu en tant que seul présent face à ce qui se tient dans le temps.

40. Texte cité supra, note 19 .

41. Sentence 44, 28, passage cité supra. 
$\mathrm{La}$ «leçon» de la définition XI, formalis, complète donc la compréhension du commentaire de la définition IX secundum formam. Plus précisément, elle écarte la tentation de lire la description «méréologique» comme une allusion à la seule prédication (selon l'exemple des parties du langage qu'Aristote lui-même utilise dans Zeta 10), et conduit vers une lecture qui assigne à Dieu une place et un rôle hégémonique à l'égard de tout ce qui est en provenance de Dieu sans pouvoir se séparer de lui, alors que Dieu se tient, lui, séparé de tout, dans une transcendance sans lieu ni recours. La description «méréologique» porte donc sur cette réalité qui n’est pas formelle mais est considérée dans son actualité même, et tente de déconstruire le paradoxe d'un "présent» qui n'appartient pas au temps et d'un "tout» dont la totalité en tant qu'unité est séparée de l'étendue (du tout) et de la pluralité (des parties). Mais la description elle-même est orientée: elle assigne aussi un but à cette déconstruction, à ce dévoilement analytique du paradoxe contenu sous l'asymétrie ou la non-réciprocité entre tout et parties. Elle met en lumière la persistance d'un lien immanent qu'elle exprime à l'aide d'une figure empruntée au registre des métaphores de la puissance.

La surabondance de la dunamis - exabundanter dans la définition XI ayant vraisemblablement le même sens que la surabondance de puissance,

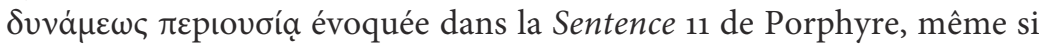
celle-ci est assignée à des réalités incorporelles et non à Dieu - confère à Dieu une prééminence qui se traduit dans le commentaire de la définition IX par l'attribution au «tout» d'une vue de type synoptique. La puissance se définit donc comme un acte, mais comme un acte de saisie, d'appréhension, non de production. Son caractère prééminent, propre à cette puissance, se confirme par la possibilité d'exercer cet acte unique, ce regard pénétrant, intuitus unicus, sur la totalité du «tout». Or, en s'exerçant ainsi, en faisant acte de la puissance qui incombe à la vision, cet acte prééminent compris dans la puissance et comme puissance, et qui ne participe pas de la production de l'ensemble des parties sans être toutefois séparé, crée à partir de lui-même ce sur quoi il s'exerce effectivement, à savoir la totalité $\mathrm{du}$ «tout» ${ }^{42}$. La totalité est donc le fruit de ce regard unique et prééminent, qui peut voir «tout» simultanément puisque ce qu'il voit comporte un certain «tout», le sien, saisi comme expression formelle de sa nature unique présente en toutes les parties. L'un se reflète comme un dans des

42. On peut aussi rapprocher cet acte propre à la puissance qui exerce sa maitrise sur ce que celle-ci est fondamentalement, des analyses que Plotin consacre à la volonté de l'Un à l'égard du Bien dans Enn. VI, 8 [Traité 39], 9 (l'image du roi qui se possède lui-même sans s'incliner devant la royauté) et 18 (le Bien «est souverainement le maître de ce qui vient après lui et, au premier chef, de lui-même», L. Lavaud (trad.), Paris, GF, 2007). 
miroirs multiples, disait Plotin ${ }^{43}$. Ainsi, ce regard ne se voit pas lui-même en saisissant la totalité, mais ne voit pas non plus ce qui serait tout autre que lui, à savoir la pluralité.

Les usages philosophiques du vocabulaire de la vision et en particulier

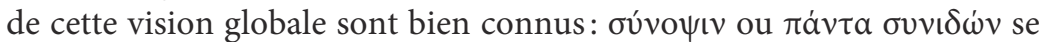
trouvent chez Platon, dans République VII (537c 2) et dans les livres IX et $\mathrm{X}$ des Lois (858c 3 et $904 \mathrm{~b} 3$ ). Bien que le contexte d'origine puisse induire un sens politique dans l'usage de la vue synoptique, la facilité de cette lecture au premier degré doit céder la place à une signification et à une visée d'un autre ordre, aussi bien chez Platon que dans le commentaire de la définition IX du Livre des définitions de Dieu. La sunopsis, caractérisant la dialectique, permet à ceux qui en possèdent l'art, d'embrasser à la fois les sciences (des réalités immuables) dans les rapports des unes envers les autres, et de surcroît la nature de l'être (la réalité effective des choses existantes) ${ }^{44}$. Corroboré avec les deux visions qui résultent de la confrontation avec la lumière et les ténèbres selon la direction du cheminement (sortie de la caverne et retour) ${ }^{45}$, ce regard, unique et distributif en même temps, actualise par une position qui n’est ni séparée ni topique et immanente à son objet, une position de prééminence à l'égard des opposés ou du multiple. Il actualise ainsi la puissance inscrite dans « tout» ce qu'il tient d'un seul coup, et réalise de ce fait ce "tout» comme ensemble puisque ce regard, unique et simultané, s'actualise comme récepteur visuel d'une entité intégrale, solidaire en tant que «tout-entier».

La prééminence du coup d'œil panoramique ne manifeste pas une domination, bien que la centralité et la hauteur par laquelle on imagine l'exercice d'une telle vision, conduisent à y voir une métaphore de la royauté, de l'hégémonie, du contrôle à partir d'un pouvoir unique, total et centralisé. Mais le regard synoptique joue ici un autre rôle. Il est stratège, juge et condition en même temps, condition par laquelle s'accomplit la

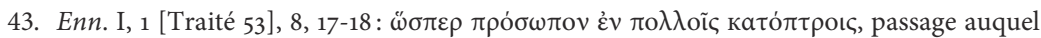
j'avais fait référence supra, note 1 . Dans un contexte différent, où l'intellect et non l'âme constitue l'objet de l'analyse, Plotin utilise le vocabulaire du regard pour désigner l'acte par lequel l'intellect regarde le Beau: «Ainsi, parce qu'il est Intellect il le regarde, et quand il le

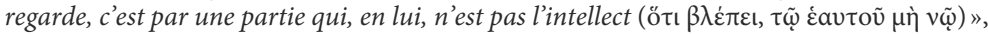
Enn. V, 5 [Traité 32], 22-23, Plotin, Traités 30-37, R. Dufour (trad.), Paris, GF, 2006; c'est moi qui souligne). Suit une topologie de l'acte qui met en lumière d'autres parallélismes avec les définitions du Liber sur lesquels il faudrait revenir un jour.

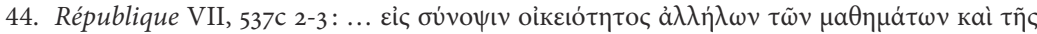

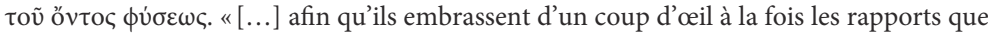
les sciences ont entre elles, et la nature de l'être», Platon, République, E. Chambry (trad.), Paris, Les Belles Lettres, 1946, p.180.

45. République VII, 518a 1-b 5. 
connaissance unique de tout ce qui se déploie. S'il est «roi», c'est nécessairement un «roi philosophe» qui exerce alors sa puissance de cette manière et dans ces conditions. Mais rien ne dit pourtant que ce regard incarne ou hypostasie le roi. Comme dans le commentaire latin de la définition IX, ce n'est pas Dieu un et seul qui regarde mais le «tout» qui embrasse de sa vue toutes les parties, ce «tout» dont Dieu est le "présent» de ce qui est dans le temps (quicquid cuius temporis est) et dont la déité correspond à la totalité. En prenant à rebours le raisonnement de la définition et de la glose latine à la lumière de l'image platonicienne du regard synoptique, on pourrait dire que la totalité signifie la déité dans sa transcendance à l'égard du «tout », et que le "présent» face au temps signifie Dieu en tant qu'instant seul et unique comprenant d'un seul tenant le temps, tout autant qu'il est séparé et prééminent face à la succession et à la division qui caractérisent le déploiement de l'être dans le temps et dans la multiplicité des étants. Du reste, pour les philosophes Dieu, comme l'Un des néoplatoniciens ou comme le roi de Platon, n'a pas un regard propre. Le regard, en revanche, tient le rôle de l'hégémon propre à chaque entité qui exerce les fonctions de l'intellect et de l'âme rationnelle et qui par conséquent voit tout: tà $\pi a ́ v \tau \alpha$ ó $\rho \tilde{\omega} v^{46}$. Chez Platon cette vue synoptique est d'ailleurs comparée

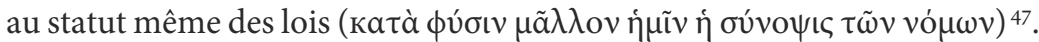
Une conformité entre notre vue de l'ensemble et la nature des lois signifie, pourrait-on dire, à la fois la possibilité d'une adéquation de l'universel à servir l'unicité de chaque être singulier, un à un, en même temps que la possibilité qui revient à chacun de reconnaître par l'exercice d'une telle vision son lien avec l'ensemble (en l'occurrence, le respect des lois). Dans un cas, cette vue panoramique est unique, par voie de synthèse, dans l'autre, elle va d'une chose à l'autre par voie de consécution et de déduction. C'est à ce regard unique que semble référer dans la glose de la définition IX la saisie globale qui s'exerce à partir du «tout» (totum... omnes partes videt), et donc la condition d'accès à la totalité à travers l'ensemble des parties successives, de même que la simultanéité par laquelle le présent se tient, comme maintenant, face au temps et hors de lui. Mais l'instant présent

46. On peut rappeler sans entrer dans le détail historiographique de la référence, cette analogie entre l'intellect-guide et la vue du tout dans De opificio mundi de Philon d'Alexandrie au sujet de l'homme créé dans un premier temps comme reflet de la pensée de Dieu. «Le rôle

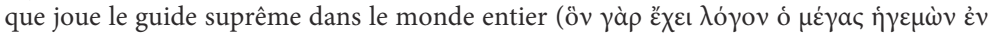

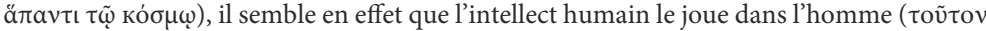

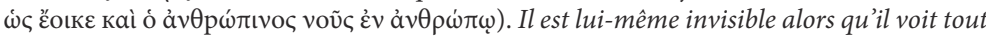

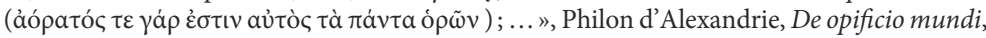
69, R. Arnaldez (trad.), Paris, Cerf, 1961, p. 186-187, c'est moi qui souligne.

47. Lois IX, 858c 2-3. 
qui comprend «tout» et qui demeure séparé de la procession immanente $\mathrm{au}$ «tout», n'a pas, ou plus précisément, ne comprend pas la connaissance du particulier, tandis que le particulier vise cet unique "présent» en vue d'une certaine connaissance dont la condition de possibilité est inscrite en lui-même.

Mais Platon, toujours dans les Lois, indique aussi un autre aspect propre à la vision synoptique, en lien direct avec le problème du «tout» $(\pi \tilde{a} v)$ et des parties: l'ordre ou la disposition dans laquelle se tient chacune des parties dans le multiple pris comme un ensemble ( $\pi \alpha \dot{v} v \tau \alpha \sigma v v ı \dot{\omega} v[\ldots]$

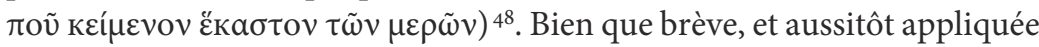
par Platon au domaine éthique (à la recherche d'une implication efficace du regard unique agissant dans la confrontation des vertus et des vices), cette indication est cependant salutaire pour saisir, au-delà de ces implications, la présence d'une conception taxinomique de la multiplicité et pour trouver donc un fondement physique sur lequel l'ordre des parties, selon qu'il est indifférent ou régi par une succession donnée, permet de structurer l'ensemble et de déterminer la nature de l'accès au «tout» soit par une pensée de l'universel, soit par une approche globale, une vision créatrice de la totalité elle-même à partir du «tout» qu'un regard unique embrasse. Aristote, à la fin du chapitre 26 de Delta ${ }^{49}$, prend appui sur le rôle joué par la position des parties pour établir la nature du tout et distinguer entre l' «ensemble» (pan) et le "tout-entier» (holon). Le premier convient au rassemblement du multiple, le second à l'entité d'un tout-entier dont l'identité est double, à la fois multiple et donnant lieu à une unité. Dans le Livre des XXIV cette distinction, de même que la question topologique et taxinomique des parties, n'intervient qu'indirectement dans le commentaire de la définition IX, à supposer que l'asymétrie des points de vue, évoquée plus haut, implique aussi une disposition des parties, chacune en lien avec l'autre et avec l'ensemble auquel toutes participent comme partie et en tant qu'unité singulière. En revanche, dans la mesure où totalité est remplacée par «communauté» dans les traités de théologie trinitaire ${ }^{50}$, la taxinomie, comme expression de la relation entre les parties, joue un rôle de premier

48. Lois X, 904b 3-c 3 .

49. Dernier paragraphe du passage cité supra.

50. Voir, en guise d'exemple pour le rapport entre "communauté» (remplaçant la totalité) et "déité », un passage du Traité sur le Saint-Esprit de Basile de Césarée: «Et de même que, en

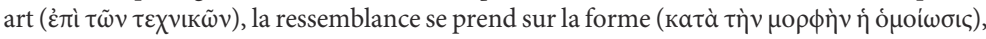

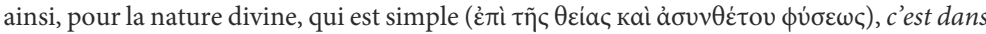

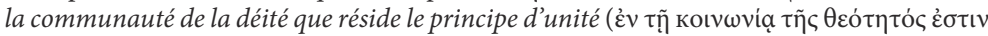

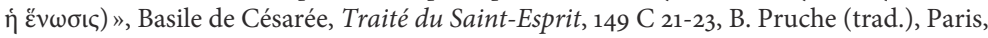
Cerf, 1947, p. 194. 
ordre. Elle sert d'appui pour la relation autodéfinissante de l'essence commune et des actions qui individualisent les hypostases, actions qui les situent les unes à l'égard des autres et qui constituent en même temps une expression spécifique du lien qui unit indéfectiblement les hypostases à travers leur réflexion réciproque. Position et ordre des hypostases prennent peu à peu le pas sur le statut des hypostases elles-mêmes dans la définition de la Trinité. Mais force est de souligner que cette question, bien que cruciale, est absente des XXIV définitions commentées du Dieu-Monade. Est-ce symptomatique de l'époque de composition de ces gloses ou révélateur de la relative discrétion de l'auteur du Liber sur les options doctrinales de sa théologie? À supposer que définitions et commentaires soient deux entités textuelles aux origines bien distinctes.

Nous ne sommes pas en mesure de répondre à cette question et donc de trancher le débat sur les origines exactes des définitions de Dieu et de leurs commentaires lapidaires qui forment le mystérieux recueil intitulé Livre des XXIV ... philosophes ou apories sur Dieu. L'ambition n'était d'ailleurs pas d'y apporter des éléments de réponse philologique ou historique, mais de reconstituer l'épaisseur théorique d'une notion, la totalité, à partir de son usage dans le commentaire de la définition IX du Liber. En inversant la perspective de la recherche, en remontant depuis cet usage particulier vers des significations plus larges, on peut en effet dessiner le contour d'un vaste domaine de la spéculation philosophique dans lequel se croisent et s'enrichissent mutuellement des thèmes de la métaphysique d'Aristote et des figures propres à la philosophie de Platon qui nourrissent la pensée et en particulier la théologie des néoplatoniciens. Placée entre les miroirs des différents textes relevant tantôt de la physique et des catégories de l'ontologie, tantôt de la noétique incluant la vision et la connaissance, la notion de totalité, jamais théorisée comme telle dans les textes anciens, émerge toutefois dans des circonstances précises où elle est appelée à répondre à un besoin spécifique de la théologie de la monade, à savoir la nécessité de réduire le multiple sans détruire la spécificité de chaque singulier.

Ainsi, la transcendance de la totalité par rapport au «tout», qui semble se dégager des différents contextes de définition et d'usage de la notion de totalité, traduit ce besoin de reconstituer et de préserver l'unicité non seulement par le mouvement double d'épanchement et de conversion, mais aussi par un acte immobile et comme donné, acte illustré ici par la prééminence de la puissance qu'exprime une vision capable d'embrasser "tout» dans la mesure où elle peut saisir la totalité que recèle chaque individu ou chaque partie. Cette capacité de saisie par un regard unique ne tient pas de la vision elle-même, qu'il s'agisse d'un organe spécifique ou du raisonnement à proprement parler, mais relève de ce qui détermine cette vision, 
de ce qui fait qu'elle puisse avoir lieu. Or la condition dont il est question ne s'identifie pas à Dieu, ne réduit pas Dieu au rôle d'une cause, mais apparaît ici à travers un acte d'abstraction illustré par le rapport de l'instant présent au temps. Le présent s'abstrait du temps puisqu'il le contient sans participer à son déploiement et donc sans jamais déroger à sa condition de présent. La saisie de la totalité par un regard unique constitue l'application logique (sous le mode «méréologique») de la définition de l'unicité propre au Dieu monos, tandis que l'abstraction ou séparation à l'égard du temps par le recours au "présent » représente de manière topique l'un des enjeux métaphysiques de la question théologique de la monade. Les deux niveaux textuels du Liber, celui de la définition et celui du commentaire, relèvent dans ce cas des deux approches de la théologie, métaphysique et logique, le commentaire utilisant les moyens de la logique pour circonscrire, voire dépasser, les apories soulevées dans chaque définition par la métaphysique ${ }^{51}$.

Anca VAsiliu

Centre Léon Robin de recherche sur la pensée antique, CNRS, Université Paris-Sorbonne

51. Je remercie Maud Pouradier de m'avoir incitée à travailler sur le Livre des XXIV en m'invitant au colloque sur la Totalité, ainsi que Carlos Steel et Pasquale Porro de m'avoir fait part de leurs doutes et critiques. 
